\title{
Improvement of Fresnel zone plate beam-profile monitor and application to ultralow emittance beam profile measurements
}

\author{
Hiroshi Sakai, ${ }^{*}$ Masami Fujisawa, Kensuke Iida ${ }^{\dagger}$ Isao Ito, Hirofumi Kudo, Norio Nakamura, \\ Kenji Shinoe, and Takeo Tanaka \\ Synchrotron Radiation Laboratory, Institute for Solid State Physics, University of Tokyo, Chiba 277-8581, Japan \\ Hitoshi Hayano, Masao Kuriki, and Toshiya Muto \\ High Energy Accelerator Research Organization (KEK), Ibaraki 305-0801, Japan
}

(Received 26 September 2006; published 30 April 2007)

\begin{abstract}
We describe the improvements of a Fresnel zone plate (FZP) beam-profile monitor, which is being developed at the KEK-ATF damping ring to measure ultralow emittance electron-beam profiles. This monitor, which is designed to have a submicrometer spatial resolution, is based on $\mathrm{x}$-ray imaging optics composed of two FZPs. Various improvements were performed to the old setup. First, a new crystal monochromator was introduced to suppress the beam image drift. Second, the two FZP folders were improved in order to realize a precise beam-based alignment during $\mathrm{x}$-ray imaging. Third, a fast mechanical shutter was installed to achieve a shorter time resolution, and an x-ray mask system was also installed to obstruct direct synchrotron radiation through the FZPs. These improvements could make beam-profile measurements more precise and reliable. The beam profiles with less than $50 \mu \mathrm{m}$ horizontal beam size and less than $6 \mu \mathrm{m}$ vertical beam size could be measured within a $1 \mathrm{~ms}$ time duration. Furthermore, measurements of the damping time and the coupling dependence of the ATF damping ring were successfully carried out with this upgraded FZP monitor.
\end{abstract}

\section{INTRODUCTION}

\section{A. Introduction to the FZP monitor}

The production of low-emittance beams is one of the key techniques for electron accelerators and synchrotron light sources. For example, a third-generation synchrotron light source and future synchrotron light sources, like an energy recovery linac (ERL), require an unnormalized emittance of a few nm rad or less (hereafter we redefine the word of "emittance" as "unnormalized emittance"). In highenergy physics, the linear collider also requires such ultralow emittance beams to realize the necessary luminosity.

The Accelerator Test Facility (ATF) was built at High Energy Accelerator Research Organization (KEK) in order to develop the key techniques of ultralow emittance beam generation and manipulation. The ATF consists of a $1.28 \mathrm{GeV}$ S-band electron linac, a damping ring, and an extraction line [1]. A low-emittance beam is generated in the ATF damping ring, where the horizontal emittance at a zero current is $1.1 \times 10^{-9} \mathrm{~m} \mathrm{rad}$. The target value of the vertical emittance at a zero current is $1.1 \times 10^{-11} \mathrm{~m} \mathrm{rad}$, which has been generated by applying precise vertical dispersion corrections and betatron-coupling corrections [2]. The typical beam sizes are less than $50 \mu \mathrm{m}$ horizon-

\footnotetext{
*Electronic address: hrsakai@issp.u-tokyo.ac.jp

†Present address: Sony Corporation, 4-3-39 Miyahara Yodogawa-ku, Osaka 532-0003, Japan.

${ }^{\ddagger}$ Present address: Laboratory of Nuclear Science, Graduate School of Science, Tohoku University, 1-2-1 Mikamine, Taihaku-ku, Sendai 982-0826, Japan.
}

tally and less than $10 \mu \mathrm{m}$ vertically. Such small beam sizes cannot be measured by the typically used visible-light imaging optics for synchrotron radiation (SR) because of the large diffraction limit of visible light. Beam-profile monitoring with good spatial resolution is crucially important to confirm whether the required extremely small emittance beam is stably generated and manipulated. Therefore, there are some special monitors set and developed in the ATF: tungsten and carbon wire scanners in the extraction line, a double-slit SR interferometer, a laser wire monitor, and a Fresnel zone plate monitor.

We briefly summarize the history of measurements of emittance in the ATF damping ring. First, the horizontal emittance was successfully measured by the tungsten and/ or carbon wire scanner set on the extraction line [3], and was also measured by a double-slit SR interferometer. However, the vertical emittance was not clearly measured with these monitors. In the case of the wire scanner, the spurious dispersion and coupling from the large horizontal emittance could be easily mixed with the vertical direction [4]. On the other hand, a vertical beam-size measurement by a double-slit SR interferometer, which used the spatial pattern of the interference of the visible SR passed through a double slit with an fixed interval [5], had an uncertainty because the measured vertical beam size in the ATF damping ring was almost at its resolution limit [6]. To avoid these uncertainties, a laser wire monitor was developed to measure directly the vertical beam size in the ATF damping ring [7]. This monitor is based on the Compton scattering of electrons with a thin laser light target, called a laser wire. 
By scanning the laser wire instead of the solid tungsten and carbon wire, quasinondestructive measurements can be performed in the ATF damping ring, and the vertical emittance was successfully measured [8-10]. Unfortunately, we could measure the beam size only in one direction. Therefore, we did not know the $x-y$ coupling of the transverse beam profile. When there is a $x-y$ coupling in the transverse beam motion, the measured vertical beam size is contaminated by the horizontal one and the beam profile will become tilted by rotating toward the original two transverse directions perpendicular to the electron-beam motion. The vertical beam size cannot be measured precisely as long as the tilt of the beam profile caused by the $x-y$ coupling is unknown. Furthermore, it takes several minutes to finish the measurement of the one directional beam size by the laser wire monitor. Thus, the effects of the beam drift and/or the mechanical vibration, which excites the beam motion of the same order as vertical beam size, cannot be removed off during the vertical beam-size measurement by the laser wire monitor. For precise beam-profile monitoring, it is necessary to know the beam image, which has much information about not only the horizontal and vertical beam sizes, but also the beam positions, beam current, tilt of the beam profile caused by the $x-y$ coupling, and its distribution, by direct monitoring of the beam image in a short time. This situation led us to develop a new beam-profile monitor based on x-ray imaging optics by using Fresnel zone plates (FZPs).

The beam-profile monitor with $\mathrm{x}$-ray imaging optics will allow precise and direct beam imaging in a nondestructive manner because the effect of the diffraction limit can be neglected by using x-ray SR. Some beam-profile monitors based on the $\mathrm{x}$-ray imaging optics were performed by using FZP and a refractive $x$-ray lens $[11,12]$. However, they used a knife-edge scanning technique to measure the beam profile because the beam image was reduced by using only one FZP or a single refractive x-ray lens. Therefore, it took a long time to measure a beam profile. In order to overcome this defect, we proposed a real-time beam-profile monitor based on magnified $x$-ray imaging optics using "two" FZPs (hereafter called as "FZP monitor") [13]. We originally developed the FZP monitor in the ATF damping ring to measure a small beam profile. For this purpose, the spatial resolution of this monitor was designed to be less than $1 \mu \mathrm{m}$. With this FZP monitor, we succeeded to obtain a clear electron-beam image enlarged by 20 times with two FZPs on an x-ray CCD, and measuring an extremely small electron-beam size of less than $10 \mu \mathrm{m}$ [14]. Recently, a beam-profile monitor using a single FZP and an x-ray zooming tube has been developed at the SPring- 8 storage ring [15]. In this monitor, the magnified beam image was also obtained by using an $\mathrm{x}$-ray zooming tube, where $\mathrm{x}$ rays were converted to photoelectrons before magnification. It has a small spatial reso- lution of $4 \mu \mathrm{m}$. With this monitor at the SPring-8 storage ring, x-ray images of the electron beam were clearly obtained, and the vertical beam size with $14 \mu \mathrm{m}$ in root mean square (rms) was successfully measured with a $1 \mathrm{~ms}$ time duration.

\section{B. Motivation for the improvements}

There are several motivations for the improvements. First of all, to obtain long-term position stability of the beam image on an x-ray CCD, a new $\mathrm{Si}$ monochromator was installed to suppress the beam image drift. Second, the effect of aberrations due to a misalignment of the x-ray imaging optics was not estimated and compensated in the FZP monitor because the center of x-ray SR could not be detected with the x-ray CCD camera by intercepting the FZPs with fixed folders. The measured beam profile with the FZP monitor was sometimes deviated from a Gaussian distribution. However, we could not conclude whether the observed non-Gaussian character was caused by instrumental aberration or was a real feature of the beam itself. To eliminate the aberrations of the $\mathrm{x}$-ray imaging optics due to misalignment, two FZP folders were newly installed. Third, penetrated $x$ rays through two FZPs, which appeared on the X-ray CCD as a square of $3 \mathrm{~mm} \times 3 \mathrm{~mm}$ reflecting the FZP structure, is one of the background components of the FZP monitor and affect the beam-profile measurement. In order to reduce this background, an X-ray mask system has been installed. Finally, we improved the time resolution for reducing any short-term movements of the monitor and/or beam oscillation. Especially the AC line noise might affect the beam oscillation. In the original setup of the FZP monitor, the minimum exposure time was $20 \mathrm{~ms}$; therefore, the obtained beam images with different centers might be superposed by the AC line noise effect on the x-ray CCD. In order to remove the effect of short-term movement, like AC line noise and perform precise beam-profile measurements, a new fast mechanical shutter with a shorter exposure time was installed.

This paper is organized as follows. In the next section, we briefly present the principle of the FZP monitor. In Sec. III, we show the experimental setup of the FZP monitor, especially the improvements. Some measurement results by using the improved FZP monitor are shown in Sec. IV. The last section is devoted to conclusions.

\section{PRINCIPLE OF FZP MONITOR}

In this section, we briefly summarize the theoretical aspects of the FZP monitor and its resolution. (See Ref. [14] for details.)

\section{A. X-ray imaging optics}

The principle of a beam-profile measurement is as follows. The FZP monitor is based on the $\mathrm{x}$-ray imaging optics with two FZPs, a condenser zone plate (CZP), and 


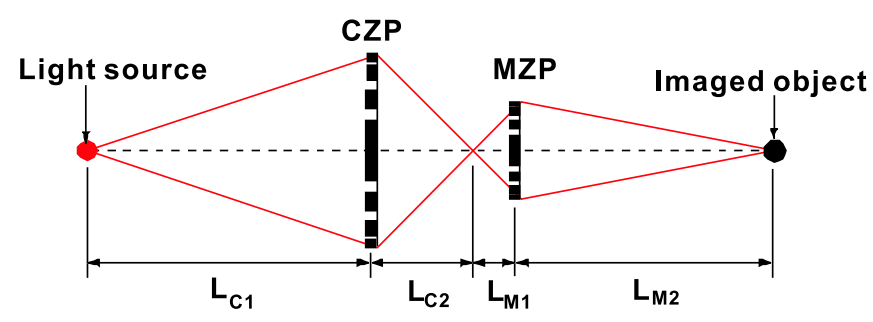

FIG. 1. (Color) X-ray imaging optics with two FZPs (CZP and MZP).

a microzone plate (MZP). It has the structure of a longdistance microscope, as shown in Fig. 1. When the electron beam emits X-ray SR light, the transverse electron-beam image is magnified on the focal plane by using this optics. The magnification, $M$, of the imaging optics is determined by $M=M_{\mathrm{CZP}} \times M_{\mathrm{MZP}}$, where $M_{\mathrm{CZP}}$ and $M_{\mathrm{MZP}}$ are the magnifications of CZP and MZP. $M_{\mathrm{CZP}}$ and $M_{\mathrm{MZP}}$ are basically defined as $M_{\mathrm{CZP}}=L_{C 2} / L_{C 1}$, where $L_{C 1}$ is the length from the SR source point to the CZP and $L_{C 2}$ is from the CZP to the intermediate focal point; $M_{\mathrm{CZP}}=$ $L_{M 2} / L_{M 1}$, where $L_{M 1}$ is the length from the intermediate focal point to the MZP and $L_{M 2}$ is from the MZP to a final focal point on an $\mathrm{x}$-ray CCD, as shown in Fig. 1. The imaging optics should be designed and optimized so that the required spatial resolution and magnification are obtained.

In the case of our monitor, we set $L_{C 1}=10 \mathrm{~m}, L_{C 2}=$ $1 \mathrm{~m}, L_{M 1}=25 \mathrm{~mm}$, and $L_{M 2}=5 \mathrm{~m}$ which resulted in $M_{\mathrm{CZP}}=1 / 10$ and $M_{\mathrm{MZP}}=200$. The total magnification $M$ was $M_{\mathrm{CZP}} \times M_{\mathrm{MZP}}=1 / 10 \times 200=20$. Then a 20 times magnified beam image was obtained on the $\mathrm{x}$-ray CCD camera in our setup.

\section{B. Fresnel zone plate}

Before estimating the spatial resolution of the FZP monitor, we introduce the fundamental characteristics of an FZP here. The radius of the $n$th zone of the FZP is expressed by

$$
r_{n}=\sqrt{n f \lambda}
$$

where the $\lambda$ is the wavelength of a photon and $f$ is the focal length for the wavelength. The spatial resolution $\delta$ which is the transverse size of a point-source image for the 1st-order diffraction on the focal plane, is determined by

$$
\delta=1.22 \Delta r_{N},
$$

where $\Delta r_{N}$ is the width of the outermost zone and the suffix $N$ means the total number of zones. If $N \gg 1$, it can be expressed as

$$
\Delta r_{N}=f \lambda / 2 r_{N}=\frac{1}{2} \sqrt{\frac{\lambda f}{N}} .
$$

The spatial resolution $\delta$ of FZP corresponds to the distance between the center and the first-zero position of the Airy diffraction pattern. When we apply a Gaussian distribution with the standard deviation $\sigma$ to its Airy diffraction pattern, the spatial resolution $\delta$ almost equals the $3 \sigma$ distance $(\delta \simeq 3 \sigma)$.

\section{Expected spatial resolution of the FZP monitor}

In order to measure a small beam profile, the spatial resolution of the FZP monitor must be smaller than the beam size. The total spatial resolution of the FZP monitor is determined by three mechanisms. One is the diffraction limit of x-ray SR light. Another is the Airy diffraction pattern of FZP, as described in Eqs. (2) and (3). The other is the pixel-size of the x-ray CCD camera, itself. We summarize the spatial resolution determined by each parameter in Table I.

The diffraction limit is determined by the wavelength of x-ray SR $\lambda=0.383 \mathrm{~nm}$, which corresponds to $3.24 \mathrm{keV} \mathrm{x}$ ray energy, and the divergence angle $\sigma_{\mathrm{SR}}$ of $126 \mu \mathrm{rad}$, which is obtained from the bending field of the ATF damping ring. Airy diffraction patterns of the FZPs also determine the limit of the measurable beam size. The image of the point source focused by each FZP has a finite width of the Airy diffraction pattern $\delta$ as shown in Sec. II B. The spatial resolution, determined by CZP (MZP), is $\sigma_{\mathrm{CZP}} / M_{\mathrm{CZP}}\left(\sigma_{\mathrm{MZP}} / M\right)$. The outermost zone widths, $\Delta r_{N}$, of CZP and MZP are listed in Table II. These $\Delta r_{N}$ 's were limited by the fabrication technology, and the outermost zone width could not be reduced by less than $100 \mathrm{~nm}$ [16]. However, we note that the effect of the Airy pattern on the spatial resolution is also less than $1 \mu \mathrm{m}$. Finally, we need to consider the pixel size of the x-ray CCD camera. When

TABLE I. Expected spatial resolution of each parameter and the total expected spatial resolution.

\begin{tabular}{lcc}
\hline \hline \multicolumn{1}{c}{ Parameters } & Definition & $\begin{array}{l}\text { Resolution } \\
(1 \sigma)[\mu \mathrm{m}]\end{array}$ \\
\hline Diffraction limit $(\lambda=0.383 \mathrm{~nm})$ & $\lambda / 4 \pi \sigma_{\mathrm{SR}}$ & 0.24 \\
Airy pattern of CZP $\left(\Delta r_{N}=116 \mathrm{~nm}\right)$ & $\sigma_{\mathrm{CZP}} / M_{\mathrm{CZP}}$ & 0.55 \\
Airy pattern of MZP $\left(\Delta r_{N}=128 \mathrm{~nm}\right)$ & $\sigma_{\mathrm{MZP}} / M$ & 0.002 \\
$\mathrm{CCD}(1$ pixel $=24 \mu \mathrm{m} \times 24 \mu \mathrm{m})$ & $\sigma_{\mathrm{CCD}} / M$ & 0.35 \\
\hline Total & & 0.7 \\
\hline \hline
\end{tabular}

TABLE II. Specifications of the two FZPs.

\begin{tabular}{lcc}
\hline \hline Fresnel zone plate & CZP & MZP \\
\hline Total number of zone & 6444 & 146 \\
Radius & $1.5 \mathrm{~mm}$ & $37.3 \mu \mathrm{m}$ \\
Outermost zone width $\Delta r_{N}$ & $116 \mathrm{~nm}$ & $128 \mathrm{~nm}$ \\
Focal length at $3.24 \mathrm{keV}$ & $0.91 \mathrm{~m}$ & $24.9 \mathrm{~mm}$ \\
Magnification & $M_{\mathrm{CZP}}=1 / 10$ & $M_{\mathrm{MZP}}=200$ \\
\hline \hline
\end{tabular}


TABLE III. Specifications of the X-ray CCD camera.

\begin{tabular}{lc}
\hline \hline \multicolumn{1}{c}{ X-ray CCD camera } & \\
\hline Type & Direct incident type \\
CCD & Back-thinned illuminated type \\
Data transfer & Full-frame transfer type \\
Quantum efficiency at $3.24 \mathrm{keV}$ & $>90 \%$ \\
Pixel size & $24 \mu \mathrm{m} \times 24 \mu \mathrm{m}$ \\
Number of pixels & $512 \times 512$ \\
\hline \hline
\end{tabular}

one CCD pixel is $\Delta x \times \Delta x$ square, its resolution, $\sigma_{\mathrm{CCD}}$, is $\Delta x / 2 \sqrt{3}$ in rms. Then, the measurable beam size is determined by $\sigma_{\mathrm{CCD}} / M$. In our monitor, one pixel is $24 \mu \mathrm{m} \times$ $24 \mu \mathrm{m}$ square, as shown in Table III. The spatial resolution determined by the CCD pixel size $\left(\sigma_{\mathrm{CCD}} / M\right)$ is $0.35 \mu \mathrm{m}$ in rms. The total spatial resolution is estimated from the sum of squares of these resolutions. The expected total spatial resolution of this monitor results in about $0.7 \mu \mathrm{m}$ in $\mathrm{rms}$. Submicron spatial resolution is expected for this FZP monitor.

\section{IMPROVEMENTS ON THE EXPERIMENTAL SETUP}

In this section, we present the setup of FZP monitor while concentrating on the improvements of the present setup compared to the former setup referred to as "old setup" in the following.

\section{A. Experimental layout}

Figure 2 shows the setup of the FZP monitor. SR light is extracted at the bending magnet (BM1R.27) just before the south straight section in the $1.28 \mathrm{GeV}$ ATF damping ring, where the horizontal beam size is expected to be about $50 \mu \mathrm{m}$ and the vertical beam size is expected to be less than $10 \mu \mathrm{m}$. This system consists of a Si crystal monochromator, two FZPs (CZP and MZP), and an x-ray CCD camera. The specifications of the two FZPs are summarized in Table II. A beryllium window with $50 \mu \mathrm{m}$ thickness is installed to isolate the relatively low vacuum of the monitor beam line from that of the ATF damping ring. $3.24 \mathrm{keV}$ x-ray SR light is selected by the $\mathrm{Si}(220)$ crystal monochromator with a Bragg angle, $\theta_{B}$, of $86.35^{\circ}$. The CZP and MZP are mounted on folders set on movable stages in order to align these two optical components precisely across the beam direction. Furthermore, the MZP folder can move in the longitudinal direction of the beam line to search the focal point of the MZP. The monochromatized $\mathrm{x}$-ray $\mathrm{SR}$ is precisely focused on the $\mathrm{X}$ ray $C C D$ camera by adjusting the positions of the two FZP (CZP and MZP) folders. The magnifications of the FZPs, $M_{\mathrm{CZP}}$, and $M_{\mathrm{MZP}}$, are $1 / 10$ and 200 , respectively. Therefore, an image of the electron beam at the bending magnet is magnified with a factor of 20 on the x-ray CCD camera. The specifications of the x-ray CCD camera (C4880-21, HAMAMATSU) are summarized in Table III. The data taking timing of the $\mathrm{x}$-ray CCD camera is synchronized

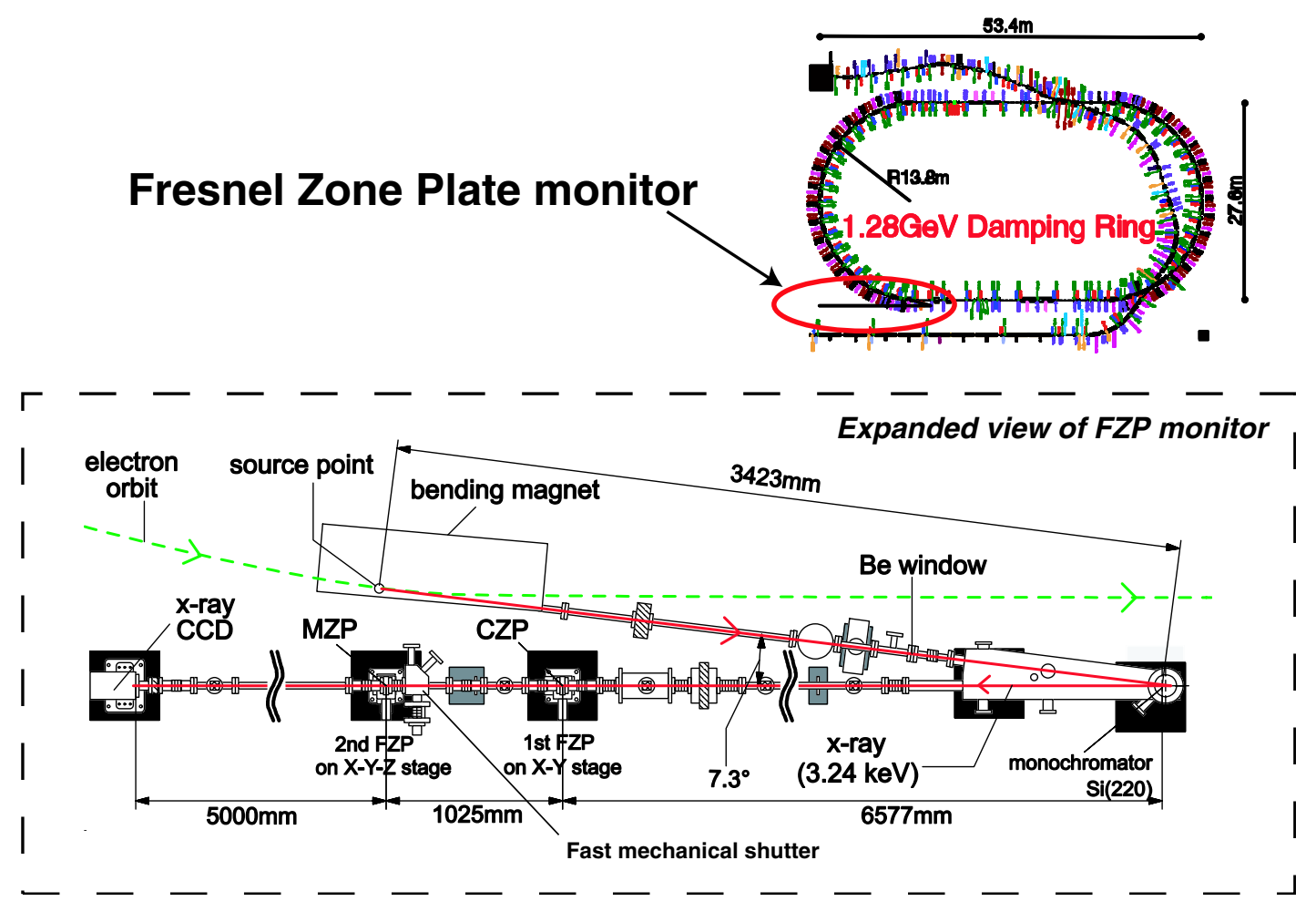

FIG. 2. (Color) Setup of the FZP monitor. 
with the beam-injection timing in order to detect a beam image during the beam operation, in which the electron beam stayed within only $500 \mathrm{~ms}$ associated with $1.56 \mathrm{~Hz}$ repetition of a beam injection in the ATF damping ring. A mechanical shutter is installed in front of the $\mathrm{X}$-ray CCD camera to avoid irradiating $\mathrm{x}$-ray $\mathrm{SR}$ on the $\mathrm{x}$-ray $\mathrm{CCD}$ camera during data transfer. The minimum shutter opening time of this shutter is $20 \mathrm{~ms}$. A new fast mechanical shutter is set between the CZP and the MZP to improve the time resolution of the FZP monitor. A detailed description of the performance is given in Sec. III D.

\section{B. Si monochromator}

The Si crystal monochromator can be rotated horizontally by using a goniometer and vertically by using a stepping motor, which is attached to the support of a $\mathrm{Si}$ crystal in a vacuum. With the old monochromator, the vertical position of the beam image on the CCD camera had largely drifted because the support of the Si crystal was deformed by heat from the stepping motor. In order to avoid any drift, a new Si crystal monochromator was produced. Figure 3 shows a picture of the new monochromator. In the new monochromator, a stepping motor was thermally isolated from the $\mathrm{Si}$ crystal by ceramic insulators and thermally stabilized by copper lines connected with a water-cooled copper plate. Figure 4 shows measurements of the beam centroid by the old and new monochromator, respectively. After this improvement, the drift was drastically reduced by a factor of about 100 and stabilized within a few $\mu \mathrm{m}$ for a long time, as shown in Fig. 4.

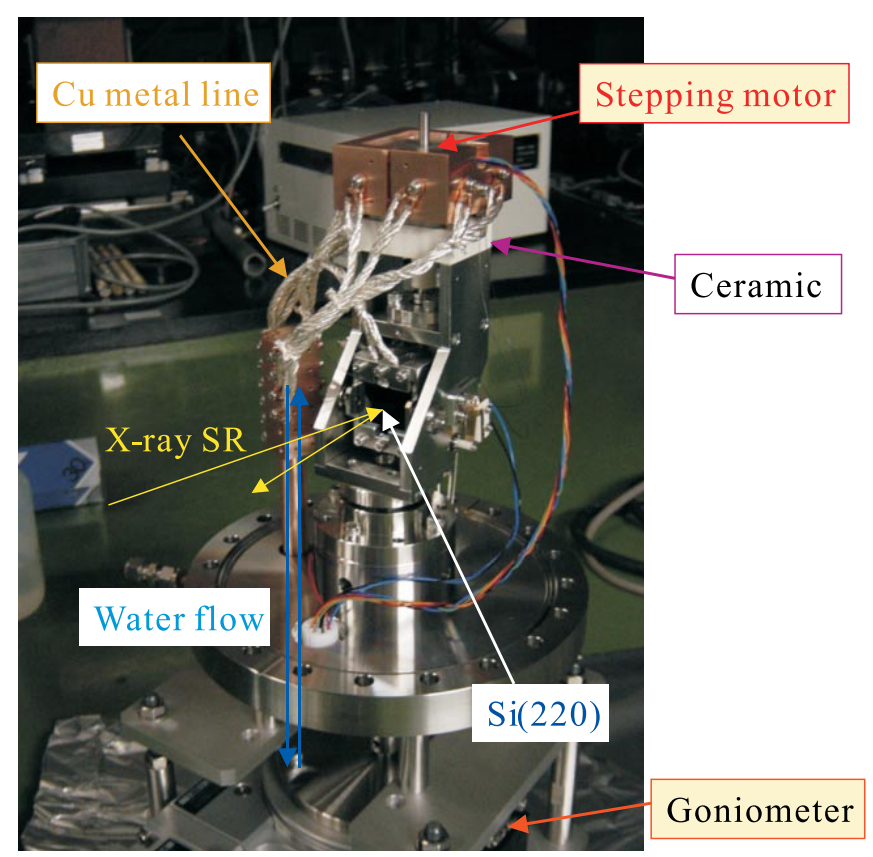

FIG. 3. (Color) Picture of the new Si monochromator.

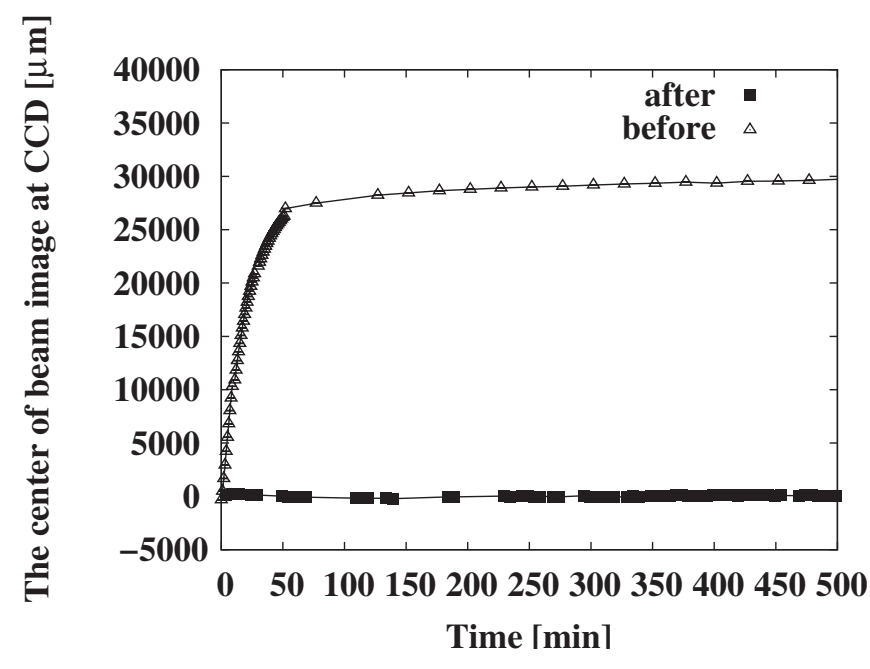

FIG. 4. Measurements of the long-term drift of the Si monochromator. The horizontal axis shows the elapsed time (minutes) and the vertical axis shows the measured vertical center of the beam image. The open triangles (solid squares) show the measured vertical center of the beam image before (after) the $\mathrm{Si}$ crystal monochromator was upgraded.

\section{Fresnel zone plate}

The new FZP folders were designed and fabricated so that the FZPs could be controlled and removed from the optical path in the vacuum if necessary. The removed FZPs are protected from the air pressure during any leaks in maintenance and repair of the monitor beam line, or the installation of new beam line components. The FZPs have never been damaged by air pressure during vacuum work since the new FZP folders were installed. Furthermore, the new folders allowed us to establish a more precise beambased alignment scheme by using only the x-ray CCD camera. A precise alignment of the FZP monitor component is crucial to avoid degradation of the spatial resolution due to aberration. The alignment procedure was greatly improved with respect to the old setup: first the center position of the x-ray beam reflected by the Si crystal (corresponding to the position of the optical axis) is measured with the $\mathrm{X}$-ray CCD without FZP imaging. After that, the CZP is inserted to the optical path and the CZP position is adjusted to the optical axis. After inserting the CZP on the optical path, a clear image of the CZP can be detected by illumination of the raw x-ray SR light, and hence the center position of the CZP can be obtained. Figure 5 shows an image of a raw $\mathrm{x}$-ray SR detected by the $\mathrm{x}$-ray $\mathrm{CCD}$ and an image of the CZP on the $\mathrm{X}$-ray CCD after inserting the CZP. The MZP position is also adjusted in the same manner. The minimum alignment error can be one pixel of the CCD $(24 \mu \mathrm{m})$ for the CZP and 1/200 (the reciprocal of the MZP magnification $M_{\mathrm{MZP}}$ ) of one pixel for the MZP. The FZP tilt angle to the optical path is decided mainly by the machining accuracy and estimated to be less than $0.5^{\circ}$. We note that the effect of these aberrations of the FZP 

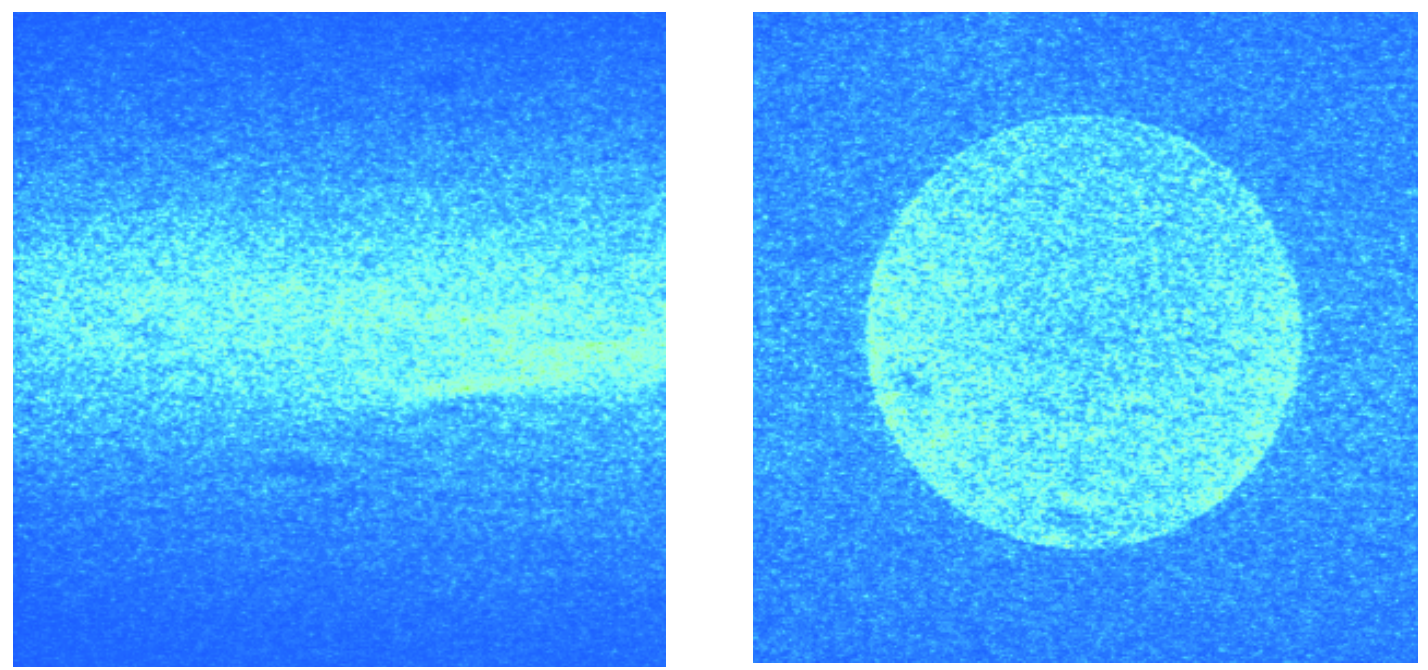

FIG. 5. (Color) (left) Image of the raw X-ray SR detected on the x-ray CCD. (right) Image of the CZP on the X-ray CCD after inserting the CZP.

monitor is calculated by not only ray-tracing analysis, but also the wave optics $[17,18]$.

\section{Fast mechanical shutter}

\section{Layout}

The time resolution of this monitor is determined by the minimum shutter opening time of the mechanical shutter. We newly install a fast mechanical shutter in the x-ray beam line in order to improve the time resolution to less than $20 \mathrm{~ms}$. Figure 6 shows a block diagram of the FZP monitor after installation. The minimum opening time of the mechanical shutter is determined by the aperture.
Therefore, we applied a fast mechanical shutter with a $1 \mathrm{~mm}$ diameter aperture (UHS1ZM2, VINCENT), which is a factor 5 smaller than the shutter located, in the old setup, in front of the $\mathrm{x}$-ray CCD camera. This shutter was installed $100 \mathrm{~mm}$ upstream of the intermediate focal point of the x-ray imaging optics between two FZPs so as not to scrape off the image of the transverse beam profile. The fast mechanical shutter was housed in a vacuum chamber and can be moved across the beam line by movable stages attached to this vacuum chamber. In addition, this shutter can be removed out from the optical path by the air cylinder if necessary.

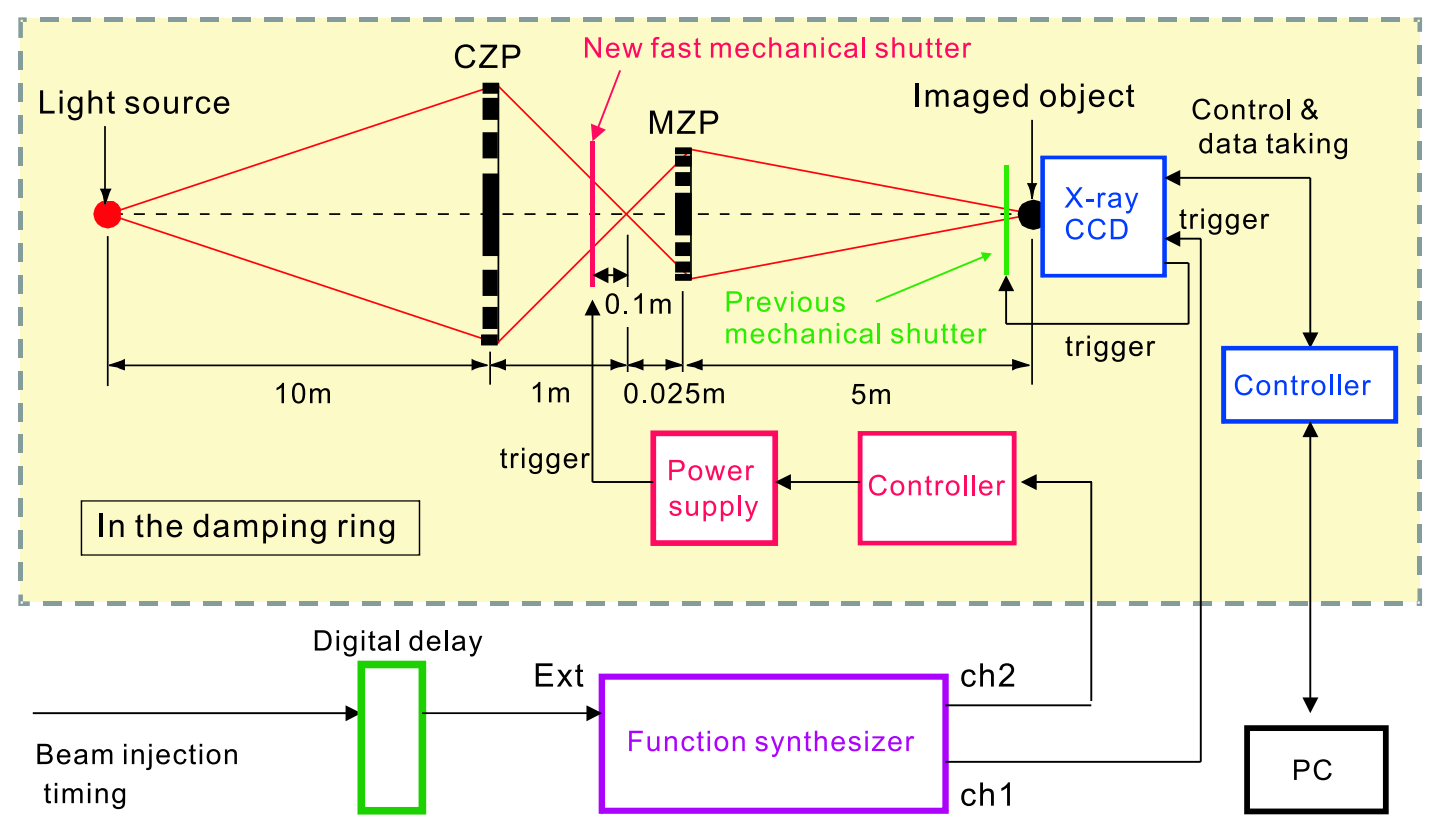

FIG. 6. (Color) Block diagram of the FZP monitor with a new fast mechanical shutter. 


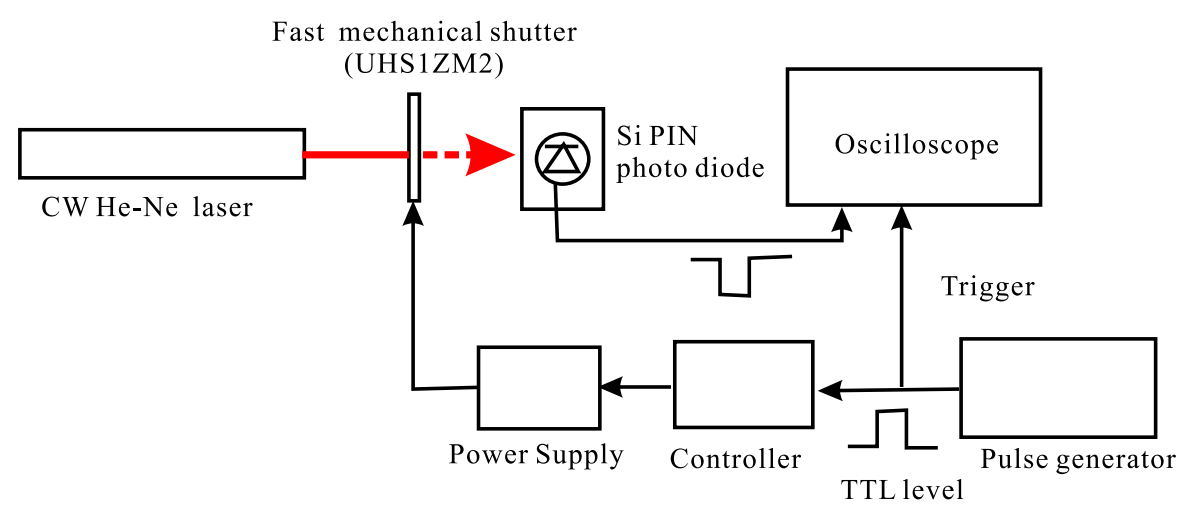

FIG. 7. (Color) Setup of a performance test of the fast mechanical shutter on a test bench.

This fast shutter has two modes. One is the "NORMAL" mode, where the shutter opening time is determined by the input transistor-transistor logic (TTL) pulse width fed into the shutter controller. Another is the "HIGH" mode, where the shutter opening time is fixed to $0.3 \mathrm{~ms}$ and synchronized with the input TTL trigger timing. These modes are changed by a shutter controller. A beam-injection timing signal is divided and provided into the CCD trigger signal ("ch1" in Fig. 6) and the shutter trigger ("ch2" in Fig. 6) via a function synthesizer. The timing of the fast mechanical shutter is synchronized with the beam-injection timing of the damping ring. The shutter opening time is controlled by changing the trigger pulse width set by the function synthesizer. Both the trigger timing and the timing delay between the fast shutter and X-ray CCD signal are also independently changed and set by this function synthesizer. On the other hand, the previous shutter trigger timing was adjusted to only the CCD internal trigger timing and controlled by the CCD controller via a PC.

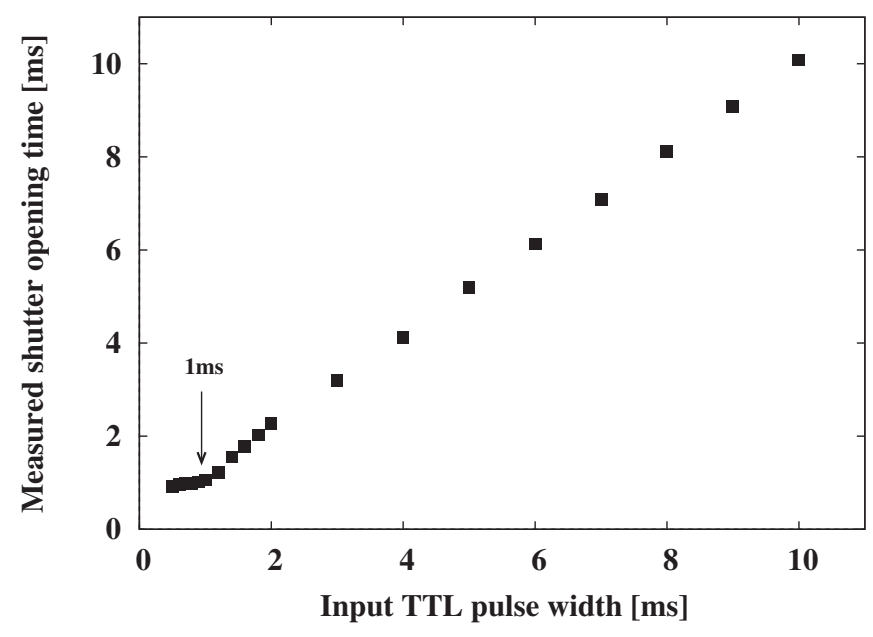

FIG. 8. Measurement results of the shutter opening time in the NORMAL mode. The horizontal axis shows the input TTL pulse width into the shutter controller. The vertical axis shows the fullwidth half maximum of the measured pulse width detected by the photodiode.

\section{Performance}

Prior to installation of the fast mechanical shutter, the performance of this shutter was measured on a test bench. The setup of the test bench is shown in Fig. 7. The shutter opening time was controlled by a TTL pulse produced by a pulse generator through the power supply and controller. Only when the shutter was open, the continuous-wave laser light through the shutter was detected by a Si $p-i-n$ photodiode. The fast mechanical shutter opening time was estimated by measuring the width of the signal of the photodiode via an oscilloscope. Figure 8 shows the measured shutter opening time in the NORMAL mode as a function of the input TTL pulse width. The measurement was performed with $1 \mathrm{~Hz}$ repetition. The opening time is given by the full-width half maximum of the measured pulse width detected by Si $p-i-n$ photodiode. The measured shutter opening time follows the input TTL pulse width down to $1 \mathrm{~ms}$, but it is saturated at less than $1 \mathrm{~ms}$ TTL pulse width. We then found that the minimum shutter opening time of this new shutter was $1 \mathrm{~ms}$ in the NORMAL mode. We also measured the shutter opening time in the HIGH mode, and the $0.3 \mathrm{~ms}$ shutter opening time was obtained. These values are consistent with the catalogue values of the minimum shutter opening time in both the NORMAL and HIGH modes. The NORMAL mode was mainly used in beam-profile measurement, unless otherwise noted. We note that this shutter performance was kept stable for at least one day in this test bench.

\section{E. X-ray pinhole mask}

The background on the CCD image mainly consists of the readout noise of the x-ray CCD circuit and the $\mathrm{x}$-ray beam transmitted through the FZPs, which is not focused at all. The transmitted $x$ rays through the MZP appear on the $x$-ray CCD as a square of about $3 \mathrm{~mm} \times 3 \mathrm{~mm}$, reflecting its MZP structure. Because the transmitted $x$ ray depended on the beam current, we needed to prepare background data of each beam current in the old setup to subtract the background component. In order to reduce the background component of the transmitted $\mathrm{x}$ ray, an x-ray mask system 

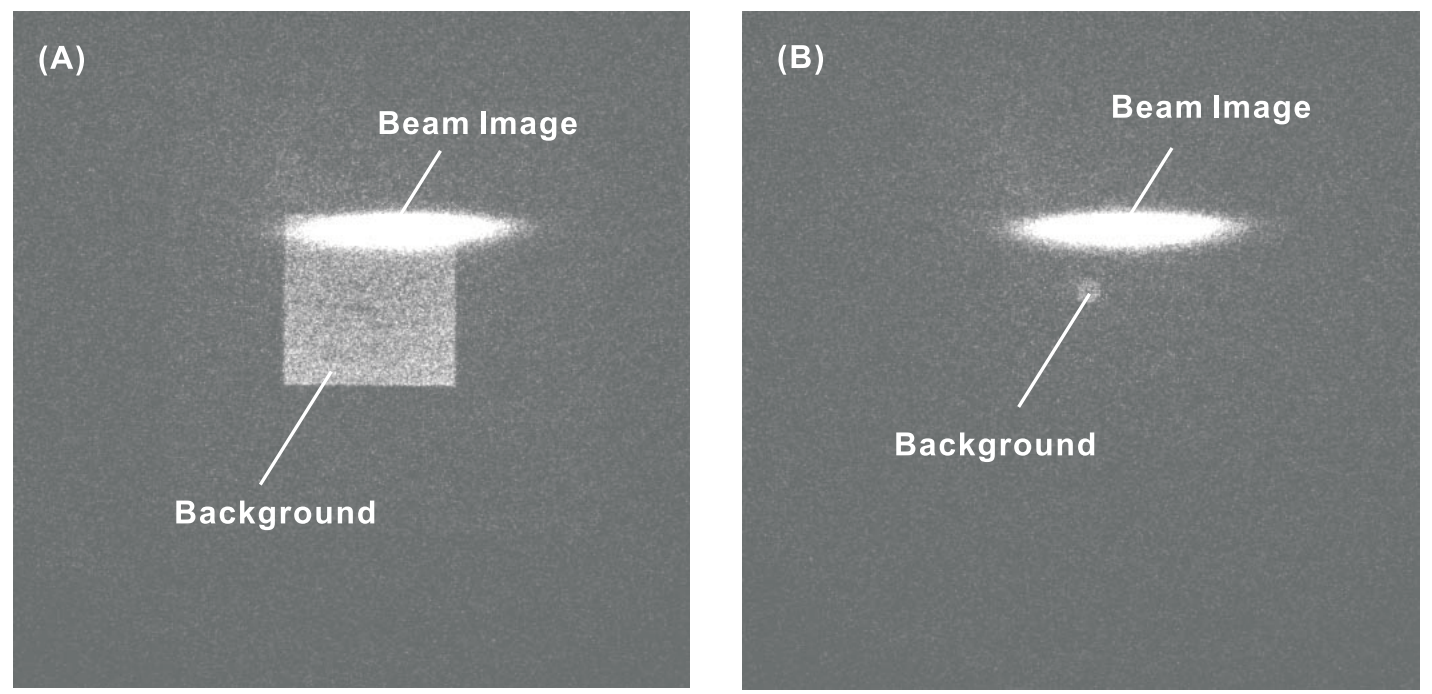

FIG. 9. CCD images of the beam profile and background (A) before and (B) after insertion of the $\mathrm{x}$-ray mask with a pinhole diameter of $300 \mu \mathrm{m}$.

with a pinhole was installed near the fast mechanical shutter. The $\mathrm{x}$-ray mask was made of stainless steel and can be moved in the horizontal and vertical directions. Figure 9 shows the CCD images before and after insertion of the x-ray mask with the pinhole diameter of $300 \mu \mathrm{m}$. The size of the background area was greatly reduced by using this $\mathrm{x}$-ray mask.

\section{MEASUREMENT OF THE ULTRALOW EMITTANCE BEAM IN THE ATF DAMPING RING}

\section{A. Beam tuning and condition}

We obtained a data set of the beam profile mainly for three days with various damping-ring conditions after improving the FZP monitor. In all cases the ATF ring was operated at $1.28 \mathrm{GeV}$ in single-bunch mode. Typical stored beam current in the ATF damping ring is above $3.5 \mathrm{~mA}$, which corresponds to $1.0 \times 10^{10}$ electrons per bunch, during beam-profile measurements. Before the measurement, the electron beam in the ATF damping ring was tuned as follows. First, the closed-orbit distortion and verticalmomentum dispersion were reduced as much as possible. Second, the coupling between the horizontal and vertical betatron oscillations was minimized by optimizing two sets of skew magnets wound around two series of sextupole magnets, respectively. This process, called "skew correction," is a key to production of a low-emittance beam [19]. In 2005, a study of the effect by damping wigglers set on the two straight sections in the ATF damping ring was started [20]. We also studied the effect of the damping wigglers on the damping time by using the improved FZP monitor. The measurement dates and beam conditions are listed below. (i) A skew correction was carefully carried out to make the vertical emittance as small as possible. The damping wigglers were turned off. We examined the basic performance of the upgraded FZP monitor and measured the beam profile. The damping time was also measured $(2005 / 4 / 8)$.

(ii) The damping wigglers were turned on. A skew correction was also carefully carried out. The damping time was measured with the damping wigglers (2005/4/13).

(iii) The damping wigglers were turned off. The skew magnets were toggled to measure the beam profiles on the different coupling conditions after a skew correction was carefully carried out $(2005 / 6 / 1)$.

In order to show the beam conditions for these three days in detail, we summarize the parameters of the ATF damping ring for three days in Table IV. The measurements of only the relevant parameters will be briefly given. The $\beta$ function at source point of the FZP monitor, where $\mathrm{x}$-ray SR was emitted, was measured as follows. By changing the strength of a quadrupole near the source point, we measured any change of the betatron tune with beam position monitors (BPMs). This tune variation is related to the $\beta$ function at the quadrupole magnet. We measured the $\beta$ functions at three quadrupole magnets and these $\beta$ functions were used to evaluate the $\beta$ function at the source point with the help of a ring's lattice model. Dispersions $\eta_{x}$ and $\eta_{y}$ were measured directly by monitoring the change of the beam image center related to the change of the rf frequency. The measured vertical dispersions were less than $\pm 1.3 \mathrm{~mm}$. The momentum spread $\sigma_{p} / p$ was measured by observing the electron-beam size with a screen monitor at the extraction line, where the dispersion was very large [3]. Because of the worse beam tuning and condition at the extraction line, we could not measure the 
TABLE IV. Summary of the parameters of the damping ring (measured value). The $x(y)$ in the second column of parameters denotes the horizontal (vertical) direction of each parameter (Tune, $\beta$ function, and dispersion function).

\begin{tabular}{|c|c|c|c|c|}
\hline Parameters & & $2005 / 4 / 8$ & $2005 / 4 / 13$ & $2005 / 6 / 1$ \\
\hline Energy $(\mathrm{GeV})$ & & & 1.28 & \\
\hline rf frequency $(\mathrm{MHz})$ & & & 714 & \\
\hline Harmonic number & & & 330 & \\
\hline Momentum compaction & & & $2.14 \times 10^{-3}$ & \\
\hline \multirow[t]{2}{*}{ Tune } & $x$ & 15.186 & 15.217 & 15.177 \\
\hline & $y$ & 8.540 & 8.585 & 8.553 \\
\hline$\beta(\mathrm{m})$ at & $x$ & $0.339 \pm 0.012$ & $0.327 \pm 0.008$ & $0.349 \pm 0.005$ \\
\hline FZP monitor & $y$ & $4.05 \pm 0.09$ & $3.87 \pm 0.05$ & $3.25 \pm 0.08$ \\
\hline$\eta(\mathrm{mm})$ at & $x$ & $49.4 \pm 0.5$ & $42.6 \pm 1.9$ & $46.3 \pm 0.2$ \\
\hline FZP monitor & $y$ & $0.30 \pm 0.05$ & $-0.75 \pm 0.50$ & $1.23 \pm 0.12$ \\
\hline
\end{tabular}

momentum spread, except on $2005 / 4 / 8$. The measured momentum spread on $2005 / 4 / 8$ was $8.5 \times 10^{-4}$ at the beam current of $3.5 \mathrm{~mA}$. The current dependence of the measured momentum spread will be shown later.

\section{B. Measurement of beam profiles by the improved FZP monitor}

\section{Data taking procedures}

Prior to a beam-profile measurement, the following procedures were carried out to adjust the $\mathrm{x}$-ray optics in the monitor. First, in order to detect raw x-ray SR on the xray CCD camera, the two FZPs and the fast mechanical shutter in the vacuum were removed off from the optical path. After that, a new alignment method was applied, as described in Sec. III C. The fast mechanical shutter and the $\mathrm{x}$-ray mask were also inserted into on the optical path after searching the beam image and adjusting the transverse position across the x-ray beam line so that the measured beam image would not be scraped off. The shutter timing and opening time were optimized depending on the experimental conditions. Finally, the longitudinal position of the MZP was scanned in order to focus the beam image on the $\mathrm{x}$-ray CCD camera.

As the shutter opening time becomes shortened, the background component becomes larger than the peak signal of the obtained beam image. In order to measure the beam profiles precisely and analyze them in detail, we carefully subtracted this background component from the data of x-ray CCD, as follows. The transverse position of the beam image is much more sensitive, by a factor of 200 of the magnification of MZP, than a transverse change of the MZP. Thanks to the newly installed $x$-ray pinhole mask, the area of the transmitted $x$ ray, which is one of the background, is drastically reduced. Therefore, by changing the transverse position of the MZP by only a few microns vertically, the beam image does not overlap the transmitted $\mathrm{x}$ ray. The alignment error of this position change of the MZP is too small to deform the obtained beam image on the x-ray CCD by the effect of aberrations. After changing the position of the MZP, the background of $\mathrm{x}$-ray CCD is subtracted. These procedures for background subtraction allow us to measure the beam profiles easily and precisely.

Figure 10 shows a measured beam image after background subtraction. The shutter opening time was fixed with $1 \mathrm{~ms}$. A clear beam image was observed on the x-ray CCD camera. This beam image, as shown in Fig. 10, was obtained by superposing 10 different beam images with the same current and same trigger timing from beam injection after background subtraction in order to gain the signal-tonoise ratio. The horizontal and vertical beam profiles were obtained by projecting the beam image to each direction. In

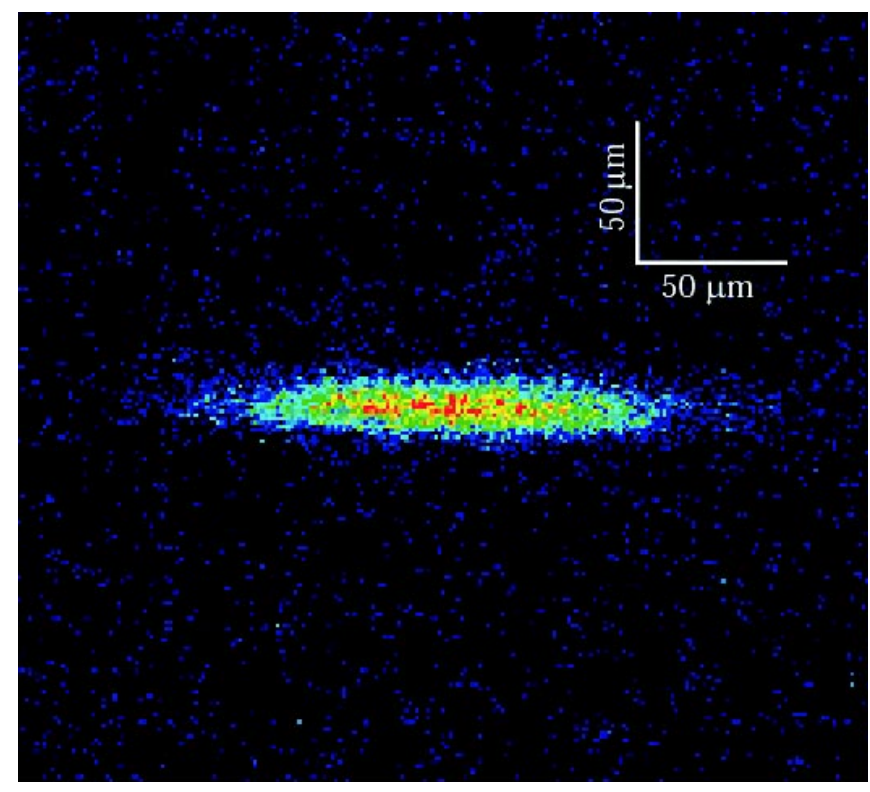

FIG. 10. (Color) Typical beam image obtained by the FZP monitor after the background was subtracted. Beam current was a $4.4 \mathrm{~mA}$ in a single-bunch mode. The shutter opening time was fixed to $1 \mathrm{~ms}$. The horizontal and vertical bars of $50 \mu \mathrm{m}$ show the scales at the source point. 


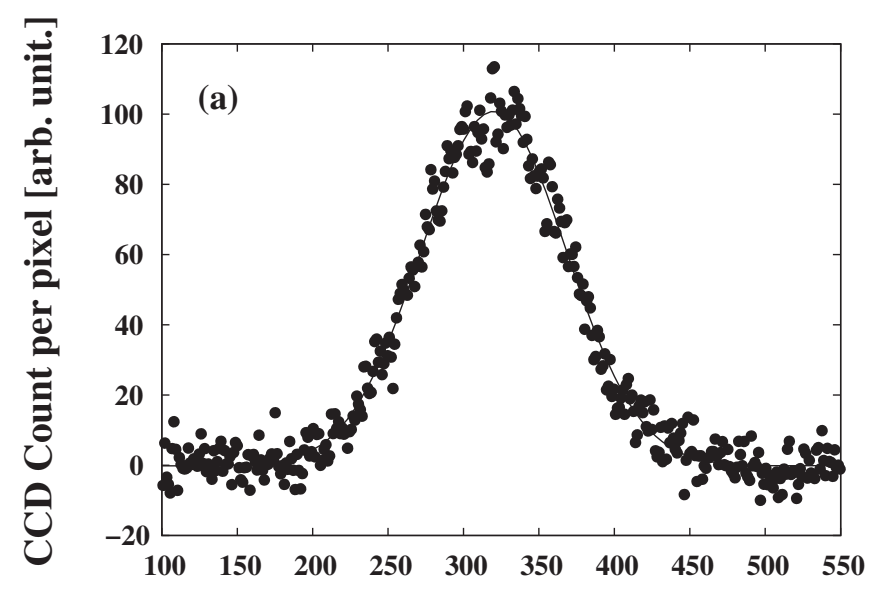

Horizontal beam position [ $\mu \mathrm{m}]$

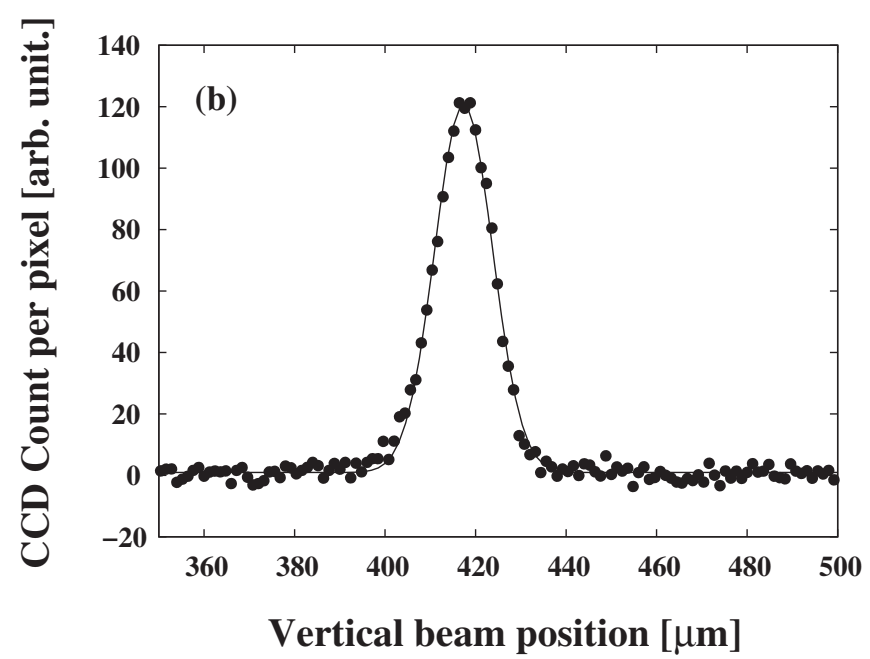

FIG. 11. Sample of (a) horizontal and (b) vertical beam profiles with their fitted Gaussian curves. The shutter opening time was fixed to $1 \mathrm{~ms}$. Data are from 2005/6/1.

order to determine the horizontal and vertical beam sizes, we fitted each beam profile to a Gaussian curve with four parameters of its center, width, peak height, and offset. The horizontal and vertical beam profiles and their fitted Gaussian curves are shown in Fig. 11. The horizontal axes in Fig. 11 show the beam positions, which were converted to the scale at the source point by dividing the magnification factor $M=20$. The measured horizontal and vertical beam sizes were about $50 \mu \mathrm{m}$ and about $6 \mu \mathrm{m}$ for three measurements.

\section{Shutter opening time dependence of the measured beam size}

The measured beam size can be increased by mechanical vibrations and/or jitter coming from electrical noises. In order to study these effects, we measured the dependence of the beam sizes on the shutter opening time prior to a precise beam-profile measurement. Figure 12 shows the measured horizontal and vertical beam sizes as a function
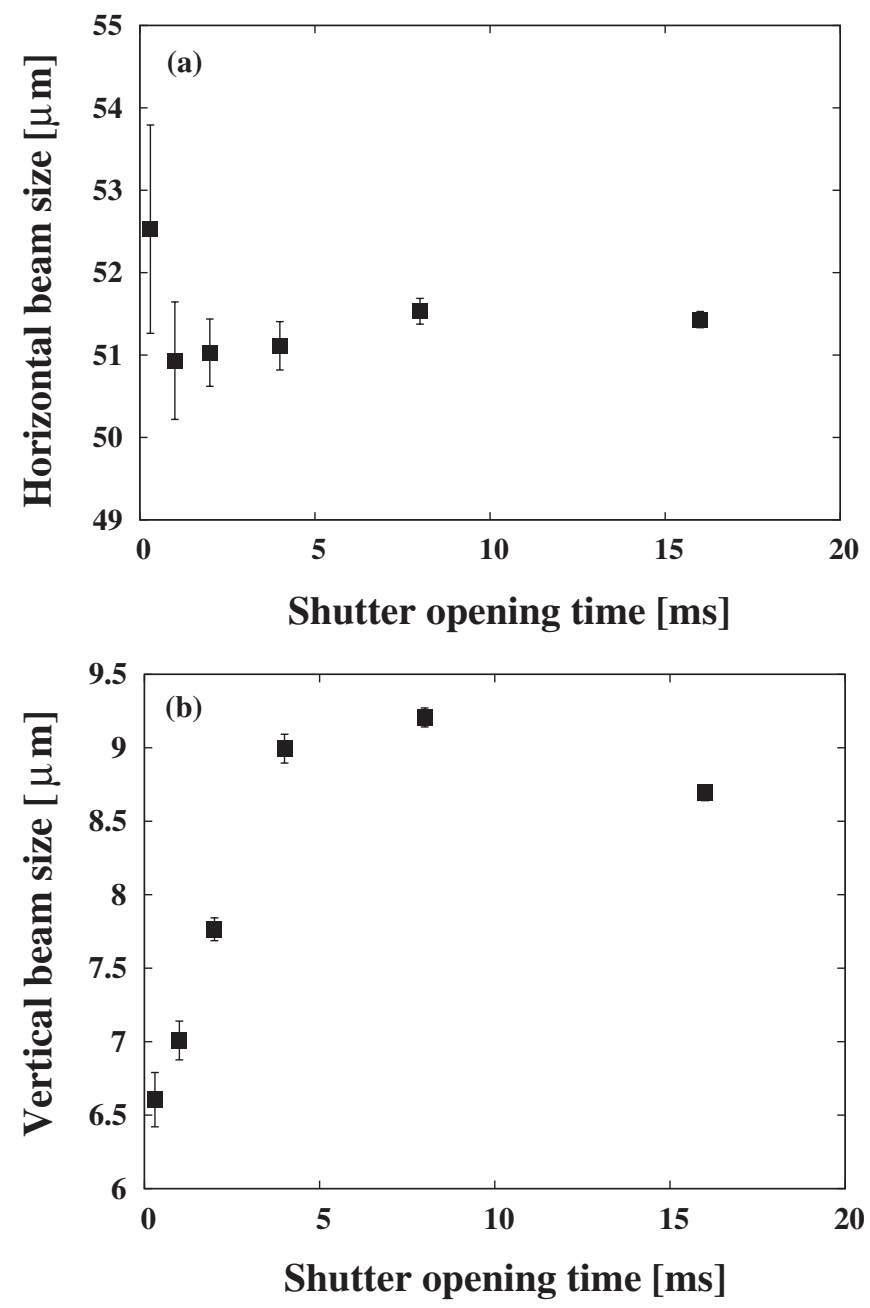

FIG. 12. Solid squares showing the measurement of the (a) horizontal and (b) vertical beam sizes as a function of the shutter opening time. The beam current was fixed at $4 \mathrm{~mA}$ in a singlebunch mode. Data are from 2005/4/8.

of the shutter opening time. The beam current was fixed to $4 \mathrm{~mA}$ during these measurements. The error bar was mainly due to the fitting error assuming a Gaussian, as described in Sec. IVB 1. We found that the measured horizontal beam size was almost $50 \mu \mathrm{m}$, and was independent of the shutter opening time. On the other hand, the vertical beam size was changed from 9 to $7 \mu \mathrm{m}$ by shortening the shutter opening time from 4 to $1 \mathrm{~ms}$ or less. In order to investigate this enhancement, we measured the image center of the CCD as a function of the trigger time of the fast mechanical shutter from beam injection with a fixed opening time. Figure 13 shows the dependences of the measured horizontal and vertical beam image centers as a function of the shutter trigger time with $1 \mathrm{~ms}$ shutter opening time. All of the data were obtained in the radiation equilibrium of the ATF damping ring. We found the horizontal and vertical oscillations of the beam position. Both oscillations were well fitted to a sinusoidal curve with $100 \mathrm{~Hz}$ frequency. We note that the position oscillation 


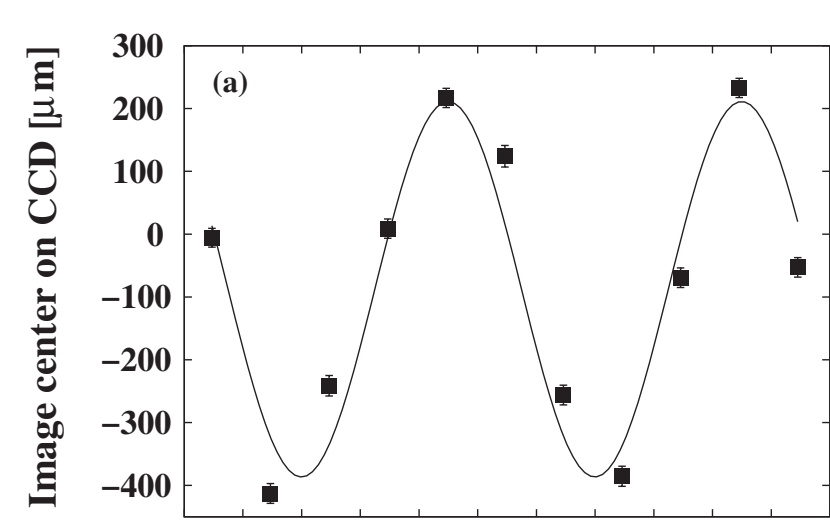

250252254256258260262264266268270272

Time from injection [ms]

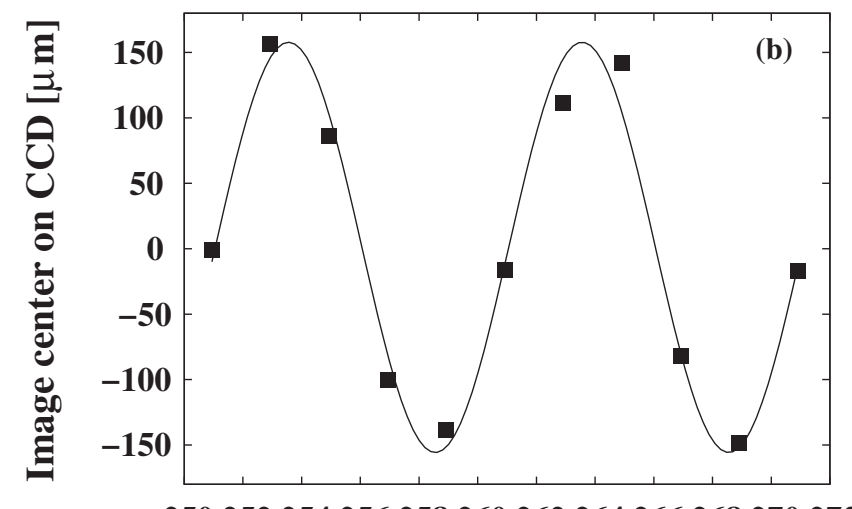

Time from injection [ms]

FIG. 13. Dependences of the measured (a) horizontal beam image center and (b) vertical beam image center corresponding to the shutter trigger time from beam injection when the shutter opening time was fixed to $1 \mathrm{~ms}$. Data are from $2005 / 4 / 8$.

was also found at $400 \mathrm{~ms}$ after beam injection with the same amplitude and the same phase of $100 \mathrm{~Hz}$ frequency. Furthermore, when we measured the beam profiles 10 times with the same shutter trigger timing, this oscillation was reproduced within these error bars. These results mean that the image center was clearly oscillating with the $100 \mathrm{~Hz}$ frequency in the synchronization with the injection timing. To translate the image center oscillation to the beam oscillation, with amplitude $A_{b}$, the sinusoidal function was magnified with the magnification factor $M=20$ of the FZP monitor. These beam oscillation amplitudes were found to be $A_{b}=14.9 \pm 1.6 \mu \mathrm{m}$ horizontally and $A_{b}=7.84 \pm 0.45 \mu \mathrm{m}$ vertically. The vertical beam oscillation significantly affects the beam size because the vertical oscillation is of the same order as the vertical beam size. From these measurements, we concluded that the vertical $100 \mathrm{~Hz}$ oscillation caused a vertical beam-size enhancement.

This $100 \mathrm{~Hz}$ oscillation was also found from data taken on 2 other different days with almost the same amplitudes and phases. In order to eliminate the $100 \mathrm{~Hz}$ oscillation from the measurement, we fixed the shutter opening time to $1 \mathrm{~ms}$ and adjusted the shutter trigger timing to an optimum phase condition of the $100 \mathrm{~Hz}$ oscillation in all of the beam-profile measurements. We note that we did not use the HIGH mode with a 0.3 ms shutter opening time from the view point of increasing the signal-to-noise ratio. Furthermore, whenever we measured the beam profiles by the FZP monitor, we superposed ten beam profiles on the $\mathrm{x}$ ray $C C D$ in order to increase the signal-to-noise ratio more.

In order to survey the source of $100 \mathrm{~Hz}$ oscillation, we checked all of the components of the FZP monitor: two FZPs, the Si crystal monochromator, and the x-ray CCD. First, mechanical vibrations of the two FZPs, including their folders, were measured at frequencies below $120 \mathrm{~Hz}$ with a compact seismometer (VSE-15D Tokyo Sokushin Co.). Figure 14 shows the measurement results of vertical vibrations of the two FZP folders. The cumulative displacement at each frequency is defined by a square root of the displacement power spectrum integrated from the
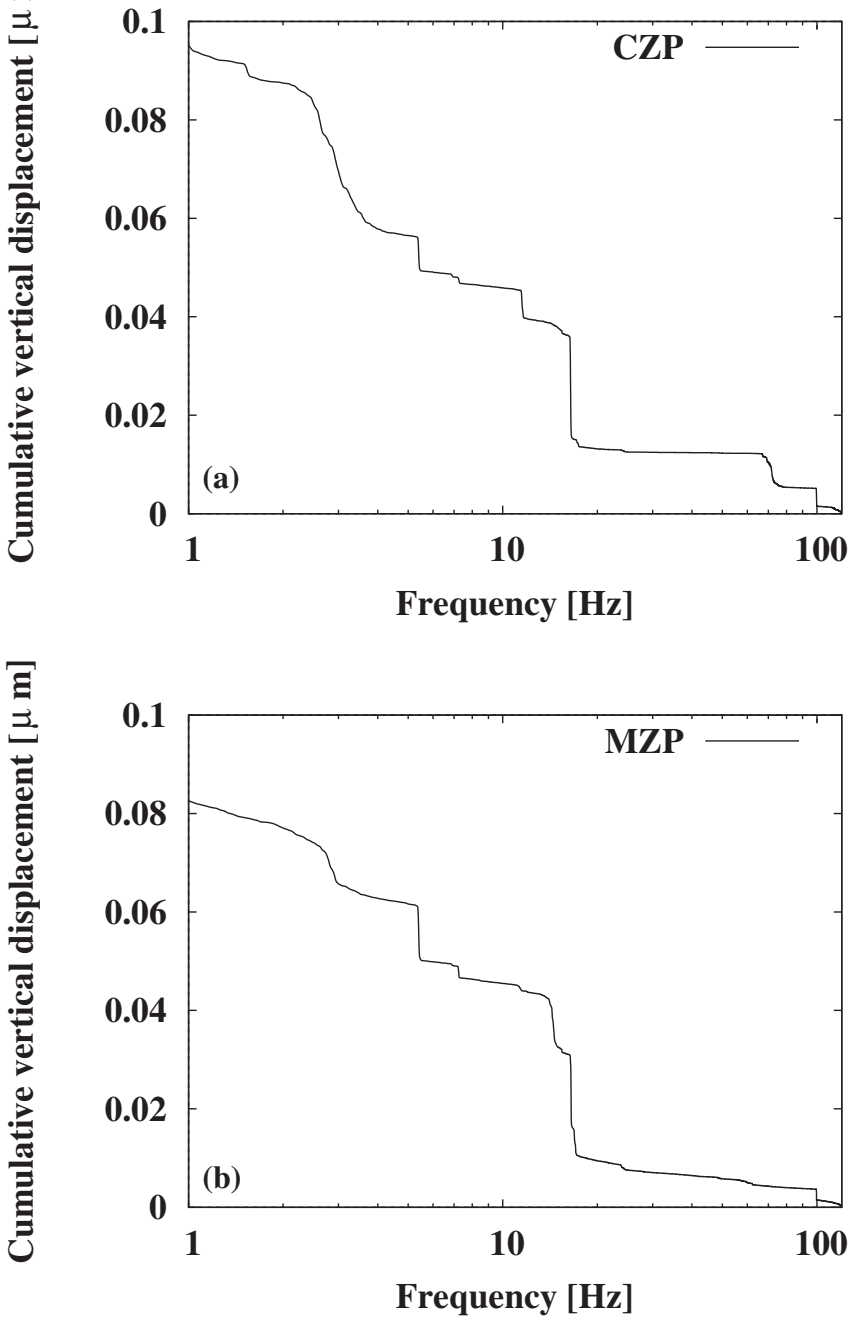

FIG. 14. Cumulative vertical displacements of (a) CZP and (b) MZP. 


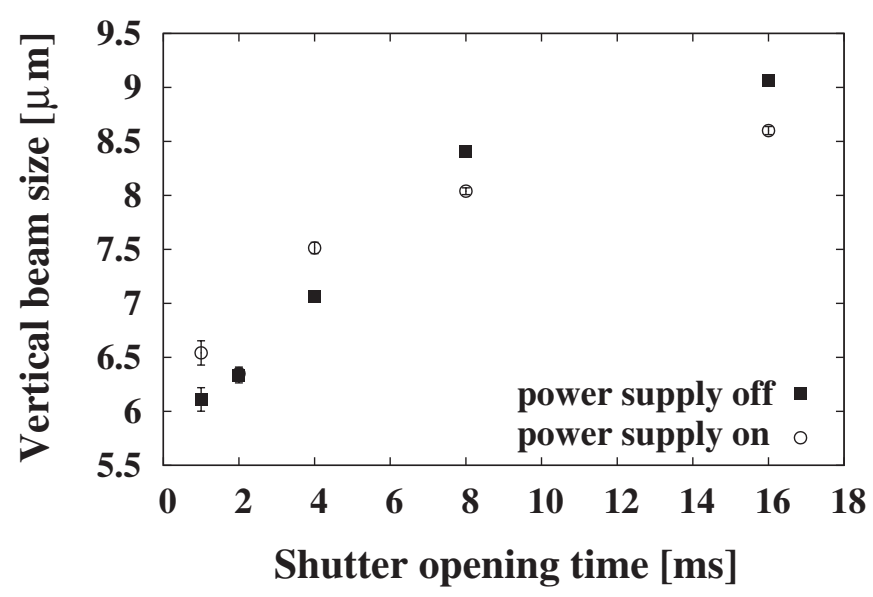

FIG. 15. Solid squares (open circles) showing the measurement of the vertical beam sizes as a function of the shutter opening time when the power supply of the Si crystal monochromator was turned off (on).

frequency up to $120 \mathrm{~Hz}$. We found a $5 \mathrm{~nm}$ rms displacement of the CZP and $4 \mathrm{~nm}$ rms displacement of the MZP at $100 \mathrm{~Hz}$ frequency, as shown in Fig. 14. The scaled displacements at the source point due to the CZP and MZP displacements of $\Delta y$, are expressed as $\left(1+1 / M_{\mathrm{CZP}}\right) \Delta y$ and $\left(1 / M_{\mathrm{CZP}}\right) \Delta y$, respectively. In our case $\left(M_{\mathrm{CZP}}=1 / 10\right.$ and $M_{\mathrm{MZP}}=200$ ), they are $11 \Delta y$ and $10 \Delta y$. Assuming the observed vibrations, 55 and $40 \mathrm{~nm}$ beam oscillations are expected by the CZP and the MZP, respectively. These values are too small to explain the $100 \mathrm{~Hz}$ beam oscillation with the vertical amplitude, $A_{b}$, of $7.84 \pm 0.45 \mu \mathrm{m}$, as shown in Fig. 13. Therefore, the vibrations of the FZPs are not the reason for the $100 \mathrm{~Hz}$ oscillation of the beam center. Second, we doubted that the Si crystal monochromator might vibrate at $100 \mathrm{~Hz}$ through its power supply of the motor and goniometer. For a confirmation, we remeasured the shutter opening time dependence when the power supply of the stepping motor and goniometer attached with $\mathrm{Si}$ monochromator were turned off. Figure 15 shows the shutter opening time dependence of the vertical beam sizes. The solid boxes (open circles) show the data when the power supply was turned off (on). We found a vertical beam-size enhancement by increasing the shutter opening time on both cases, as shown in Fig. 12. No clear difference between both conditions was observed. Finally, we measured the x-ray SR illuminated image of the CZP on the Xray CCD camera, as shown in the right picture of Fig. 5 by changing the trigger timing of the $\mathrm{x}$-ray $\mathrm{CCD}$ camera. If the beam image oscillation is due to any vibration of the $\mathrm{x}$-ray CCD camera, itself, we will also see a similar oscillation of the image of CZP because it does not follow the beam motion without focusing. We found no image oscillation of more than 1 pixel of the x-ray CCD camera horizontally and vertically, in spite of changing the trigger timing every $1 \mathrm{~ms}$ from 0 to $20 \mathrm{~ms}$ with a $1 \mathrm{~ms}$ shutter opening time.

From these measurements, we conclude that the beamsize enhancement, especially vertically, is caused by the $100 \mathrm{~Hz}$ oscillation; the FZP monitor, itself, is working well, and electron beam might be oscillated with $100 \mathrm{~Hz}$ frequency.

\section{Data analysis and results}

For data analysis, fitting with a two-dimensional Gauss function was applied to the beam images. We set 7 free parameters with horizontal and vertical centers, horizontal and vertical widths, peak height, the tilt angle, and the offset. The positive direction of tilt angle was counterclockwise to the electron-beam motion. The fitting results of the horizontal beam size $\sigma_{x}$, vertical beam size $\sigma_{y}$, and tilt angle $\theta_{b}$ are summarized in Table $\mathrm{V}$ for three different days after the skew correction. The two sets of data (named as " 1 st" and "2nd") were taken on 2005/6/1. The 1st data were taken after first making a skew correction. To confirm the reproducibility, skew magnets were turned off once, and turned on again; 2nd data on 2005/6/1 were taken under this condition. The shutter opening time was fixed at

TABLE V. Summary of the measured beam sizes, the tilt angles, and vertical emittances by the FZP monitor. A skew correction was carefully carried out.

\begin{tabular}{|c|c|c|c|c|c|}
\hline \multirow[b]{2}{*}{ Date } & \multirow{2}{*}{$\begin{array}{c}\text { Beam current } \\
I_{b} \\
(\mathrm{~mA})\end{array}$} & \multicolumn{2}{|c|}{ Beam size } & \multirow{2}{*}{$\begin{array}{c}\text { Tilt angle } \\
\theta_{b} \\
\text { (degree) }\end{array}$} & \multirow{2}{*}{$\begin{array}{c}\text { Vertical emittance } \\
\varepsilon_{y} \\
(\mathrm{pm} \mathrm{rad})\end{array}$} \\
\hline & & $\begin{array}{c}\sigma_{x} \\
(\mu \mathrm{m})\end{array}$ & $\begin{array}{c}\sigma_{y} \\
(\mu \mathrm{m})\end{array}$ & & \\
\hline$(2005 / 4 / 8)$ & 3.8 & $50.7 \pm 0.6$ & $6.50 \pm 0.07$ & $1.15 \pm 0.13$ & $10.4 \pm 0.3$ \\
\hline$(2005 / 4 / 13)$ & 3.0 & $42.5 \pm 0.7$ & $6.60 \pm 0.12$ & $2.21 \pm 0.26$ & $11.2 \pm 0.4$ \\
\hline $\begin{array}{l}(2005 / 6 / 1) \\
1 \text { st }\end{array}$ & $\begin{array}{l}4.4 \\
4.0 \\
3.5 \\
3.0\end{array}$ & $\begin{array}{l}48.2 \pm 0.4 \\
48.6 \pm 0.4 \\
48.1 \pm 0.4 \\
46.8 \pm 0.6\end{array}$ & $\begin{array}{l}6.11 \pm 0.07 \\
5.93 \pm 0.06 \\
5.94 \pm 0.06 \\
6.12 \pm 0.09\end{array}$ & $\begin{array}{l}0.70 \pm 0.09 \\
0.57 \pm 0.09 \\
0.73 \pm 0.11 \\
0.43 \pm 0.12\end{array}$ & $\begin{array}{l}11.5 \pm 0.4 \\
10.8 \pm 0.4 \\
10.9 \pm 0.3 \\
11.5 \pm 0.4\end{array}$ \\
\hline $\begin{array}{l}(2005 / 6 / 1) \\
\text { 2nd }\end{array}$ & $\begin{array}{l}4.0 \\
3.5 \\
3.0\end{array}$ & $\begin{array}{l}47.7 \pm 0.4 \\
46.4 \pm 0.5 \\
45.2 \pm 0.5\end{array}$ & $\begin{array}{l}6.03 \pm 0.07 \\
6.04 \pm 0.08 \\
6.00 \pm 0.08\end{array}$ & $\begin{array}{l}0.88 \pm 0.10 \\
1.05 \pm 0.12 \\
0.77 \pm 0.13\end{array}$ & $\begin{array}{l}11.2 \pm 0.4 \\
11.2 \pm 0.4 \\
11.1 \pm 0.4\end{array}$ \\
\hline
\end{tabular}


$1 \mathrm{~ms}$ for all data. The beam size and the tilt angle errors were mainly due to the fitting errors, so that the reduced- $\chi^{2}$ was unity. All of the beam sizes by the two-dimensional Gauss function fitting agree well with those of the onedimensional fitting on each projected direction, as described in Sec. IV B 1. All of the data show a similar current dependence, except for the data taken on $2005 / 4 / 13$. The measured horizontal and vertical beam sizes and tilt angles were distributed between 45 and $51 \mu \mathrm{m}$, between 5.9 and $6.6 \mu \mathrm{m}$, and between 0 and $1.2^{\circ}$, respectively, when the damping wigglers were turned off. When the damping wigglers were turned on, the tilt angle became larger than that without the damping wigglers. This difference might suggest that beam tuning with skew magnets was not well done when the damping wigglers were turned on.

One of our interests is to confirm the generation of the low-emittance beam, especially vertical emittance in the damping ring. The measured vertical beam size $\left(\sigma_{y}\right)$ and the vertical emittance $\left(\varepsilon_{y}\right)$ are related by

$$
\beta_{y} \varepsilon_{y}=\left(\sigma_{y}\right)^{2}-\left(\eta_{y} \frac{\sigma_{p}}{p}\right)^{2},
$$

where $\beta_{y}$ and $\eta_{y}$ are, respectively, the ring's $\beta$ function and the dispersion function in the $y$ direction at the source point, and $\sigma_{p} / p$ is the momentum spread. We note that, from Table IV, the term $\eta_{y}\left(\sigma_{p} / p\right)$ contributes less than $1 \mu \mathrm{m}$, and that it can be neglected compared with the measured beam sizes $\left(\sigma_{y}\right)$ of about $6 \mu \mathrm{m}$, as shown in Table V. Finally, we used Eq. (4) in order to evaluate $\varepsilon_{y}$; the results are listed in the 6th column of Table V. From these results, when low-emittance tuning was carefully done, the measured vertical emittance was found to be about $11 \mathrm{pm}$ rad for above $3 \mathrm{~mA}$ in the single-bunch mode.

Beam emittance in the ATF damping ring is dominated by the intrabeam scattering effect [2]. In a high current of

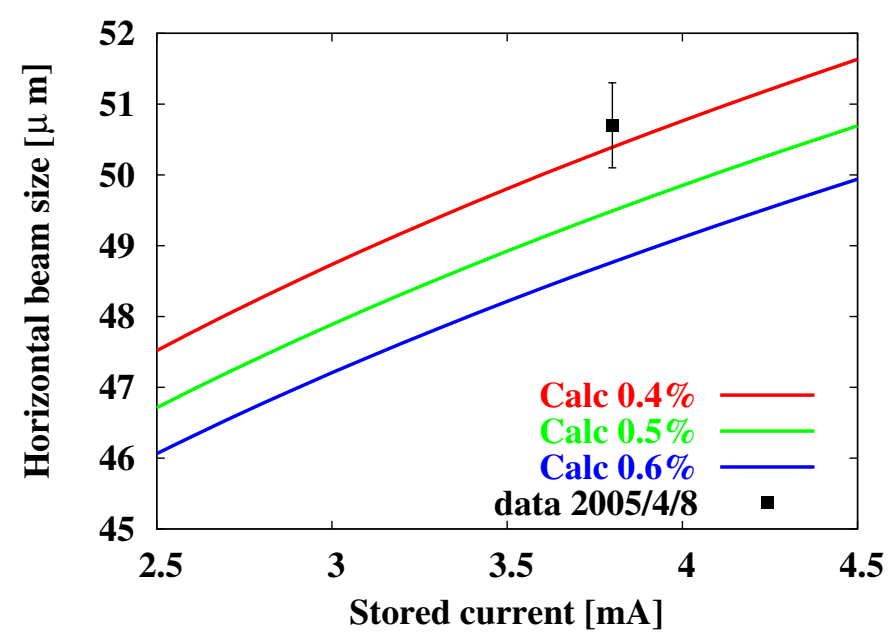

the single-bunch mode, the emittance increases as the beam current become high, and the coupling ratio decreases. In order to estimate the coupling ratio of the ATF damping ring and validate these measured beam sizes, we compared the measured beam sizes with calculation obtained by using the optics data of the ATF damping ring in Table IV and the computer program SAD [21] including the intrabeam scattering effect [22,23]. Figure 16 shows comparisons of the measured beam size (boxes) and the calculation (lines) on 2005/4/8. Figure 17 also shows the data of the measured data (boxes and circles) and calculation (lines) on 2005/6/1. The horizontal axes show the beam current of the damping ring in the single-bunch mode, and the vertical axes are the measured and calculated beam sizes. These two measurements agree well with the calculation when it is assumed that the coupling ratio is $(0.5 \pm 0.1) \%$ and are almost consistent with the other measurement by the laser wire monitor in the ATF damping ring, described in Ref. [2]. In order to confirm the assumption of coupling ratio, the energy spread was also measured. Figure 18 shows the measured energy spread at the extraction line on 2005/4/8. The errors of the measured momentum spread at higher beam current are mainly caused by the measurement error of the dispersion function at the screen monitor. On the other hand, the errors of the momentum spread at lower beam current are mainly caused by the statistical error because of the poor signal of the screen monitor. As shown in Fig. 18, the measured energy spread at the extraction line on $2005 / 4 / 8$ also agrees well with a calculation under the assumption of a $(0.5 \pm 0.1) \%$ coupling ratio.

\section{Measurement of the damping time}

By changing the trigger timing of the $\mathrm{x}$-ray CCD camera from the beam-injection timing, a beam-profile measurement during radiation damping could be carried out.

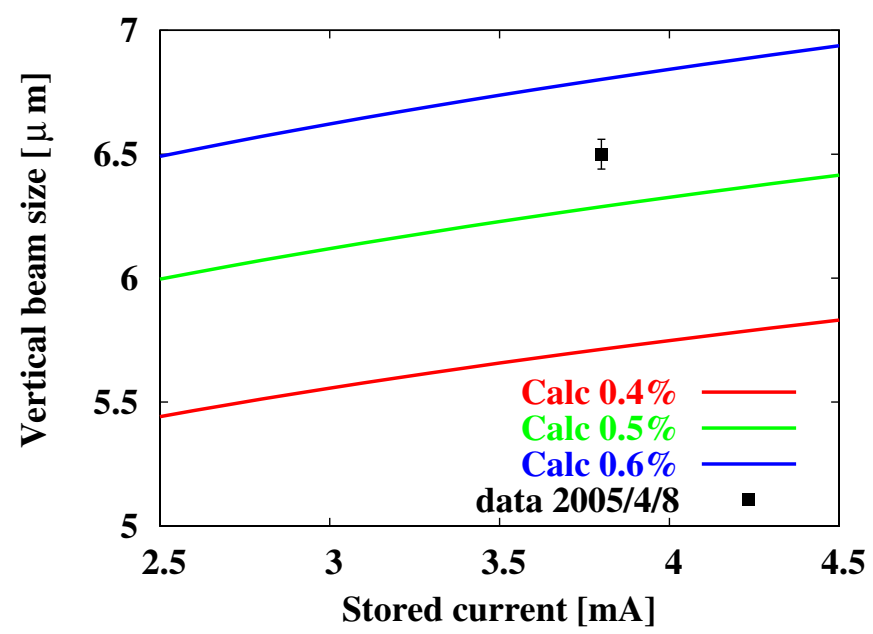

FIG. 16. (Color) Measured beam size taken on $2005 / 4 / 8$ by the FZP monitor vs beam current in the single-bunch mode. The lines are from a calculation with intrabeam scattering, assuming coupling ratios of $0.4 \%, 0.5 \%$, and $0.6 \%$. 

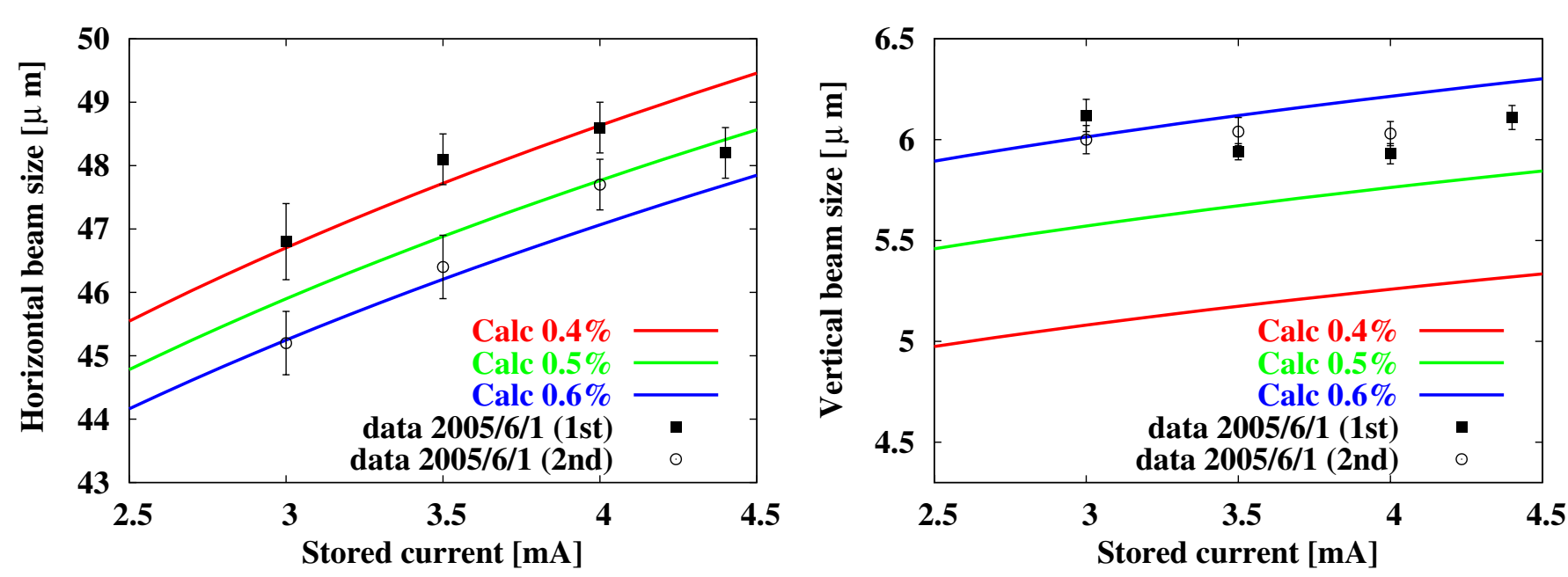

FIG. 17. (Color) Measured beam size taken on 2005/6/1 by the FZP monitor vs beam current in the single-bunch mode. The lines are from a calculation with intrabeam scattering, assuming coupling ratios of $0.4 \%, 0.5 \%$, and $0.6 \%$.

Furthermore, the improved time resolution by the newly installed fast mechanical shutter allowed us to measure the damping time. We then precisely measured the damping time with/without damping wigglers by using FZP monitor for the study of the damping wigglers.

Figure 19 shows the measurements of the damping time with/without the damping wigglers. Damping phenomena were clearly observed in the vertical direction. In order to evaluate the vertical damping time, we use the following function [24], described as

$$
\sigma_{y}=\sqrt{\sigma_{\text {inj }}^{2} e^{-2 t / \tau_{y}}+\sigma_{\text {ring }}^{2}\left(1-e^{-2 t / \tau_{y}}\right)},
$$

where $t$ is the elapsed time from injection timing. The injection beam size $\left(\sigma_{\text {inj }}\right)$, the beam size after complete damping $\left(\sigma_{\text {ring }}\right)$, and vertical damping time $\left(\tau_{y}\right)$ are used as

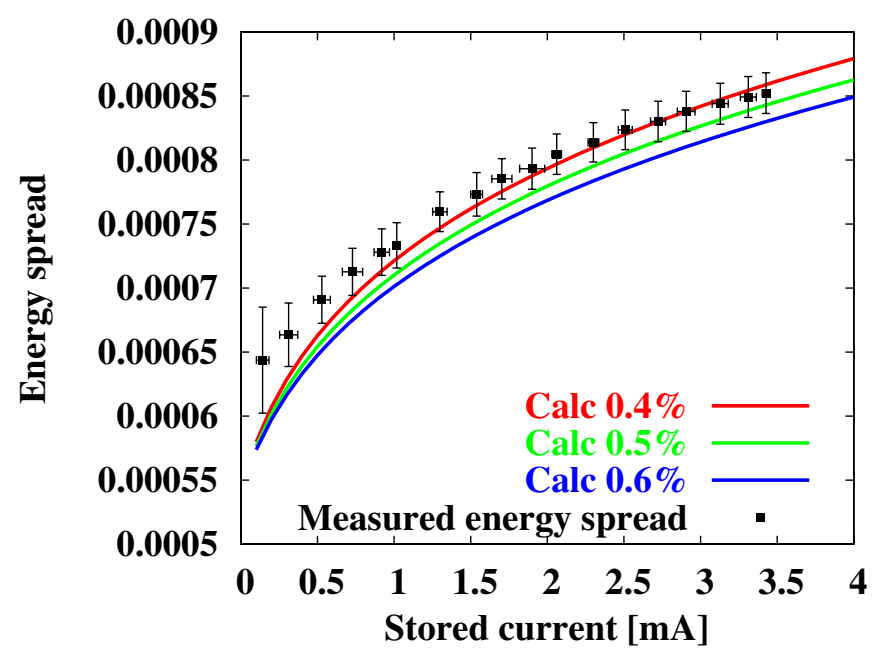

FIG. 18. (Color) Measured energy spread at the extraction line vs beam current in the single-bunch mode. The lines are from a calculation with intrabeam scattering, assuming coupling ratios of $0.4 \%, 0.5 \%$, and $0.6 \%$. free parameters for fitting. By fitting this function to the vertical beam-size measurement in Fig. 19, we obtained that the vertical damping time $\left(\tau_{y}\right)$ without damping wigglers was $(30.9 \pm 0.6) \mathrm{ms}$ and with damping wigglers $(20.7 \pm 0.8) \mathrm{ms}$. A clear difference in the measured damping time between with and without damping wigglers was observed. The design values of the damping time with/ without damping wigglers are 21.1 and $28.5 \mathrm{~ms}$, respectively. These values agree well with the damping-time measurements.

\section{Coupling dependence of the measured beam profiles}

The coupling dependences were measured by changing the currents of the skew-quadrupole coils wound on two

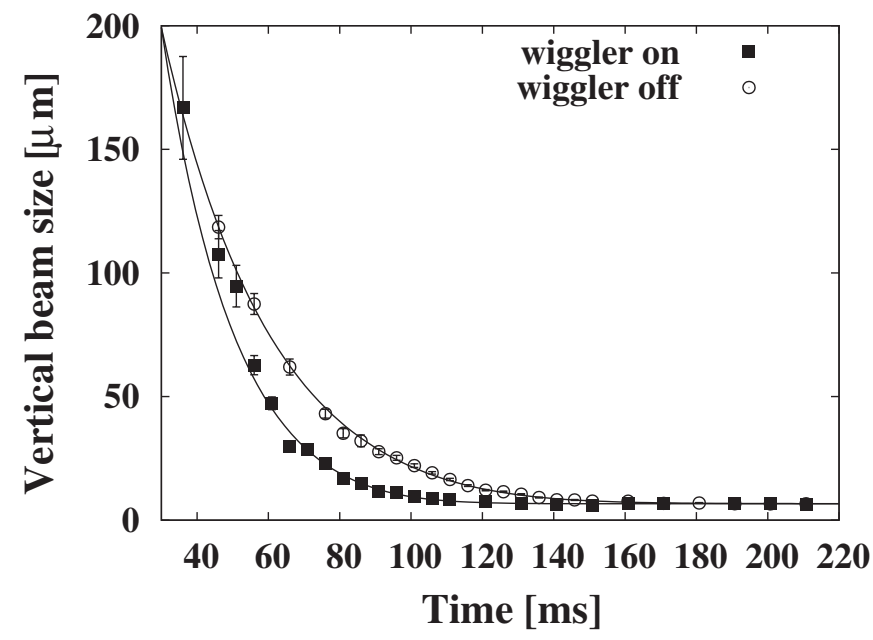

FIG. 19. Measured vertical damping time with/without the damping wigglers. The horizontal axis shows the elapsed time from beam injection into the damping ring. The vertical axis shows the measured beam size by the FZP monitor. The open circles (black boxes) show the vertical beam sizes without (with) damping wigglers. 

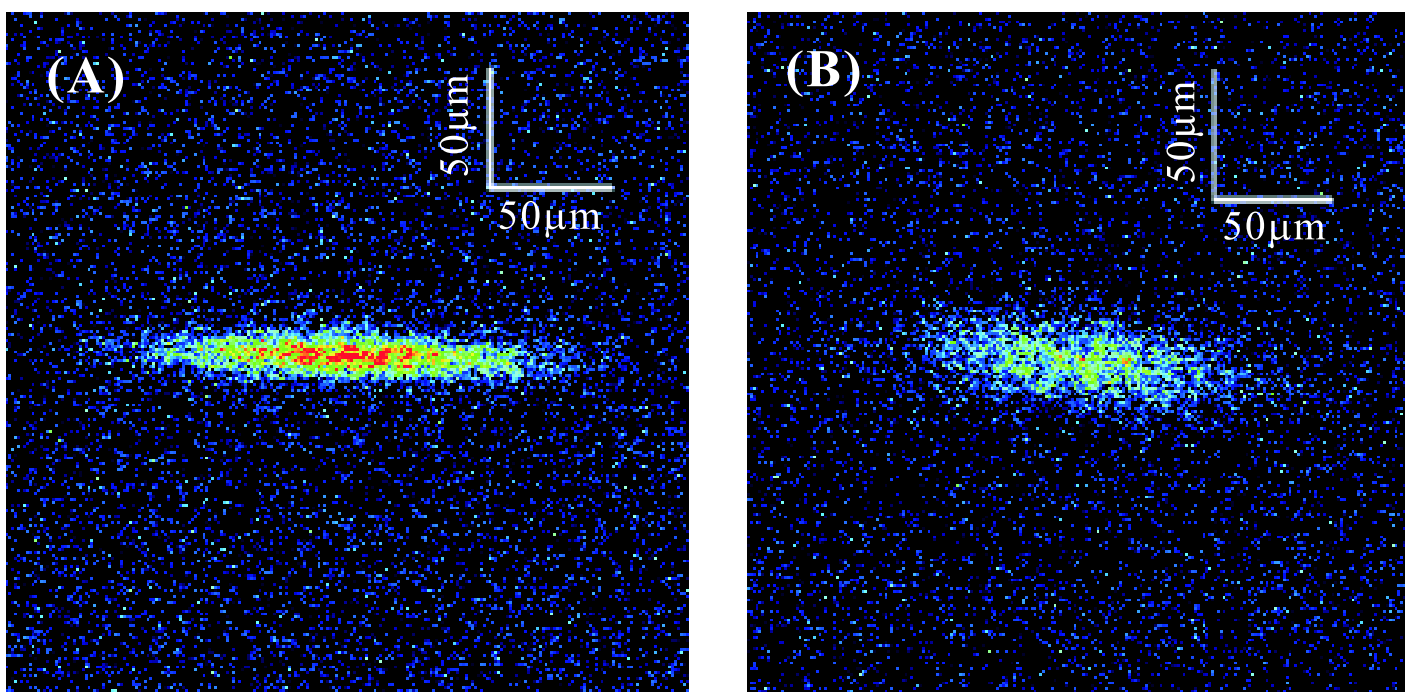

FIG. 20. (Color) Measured beam profiles when the skew correction was carefully carried out (A) and all of the skew magnets were turned off (B). The data were taken on $2005 / 6 / 1$ with a $4.4 \mathrm{~mA}$ current.
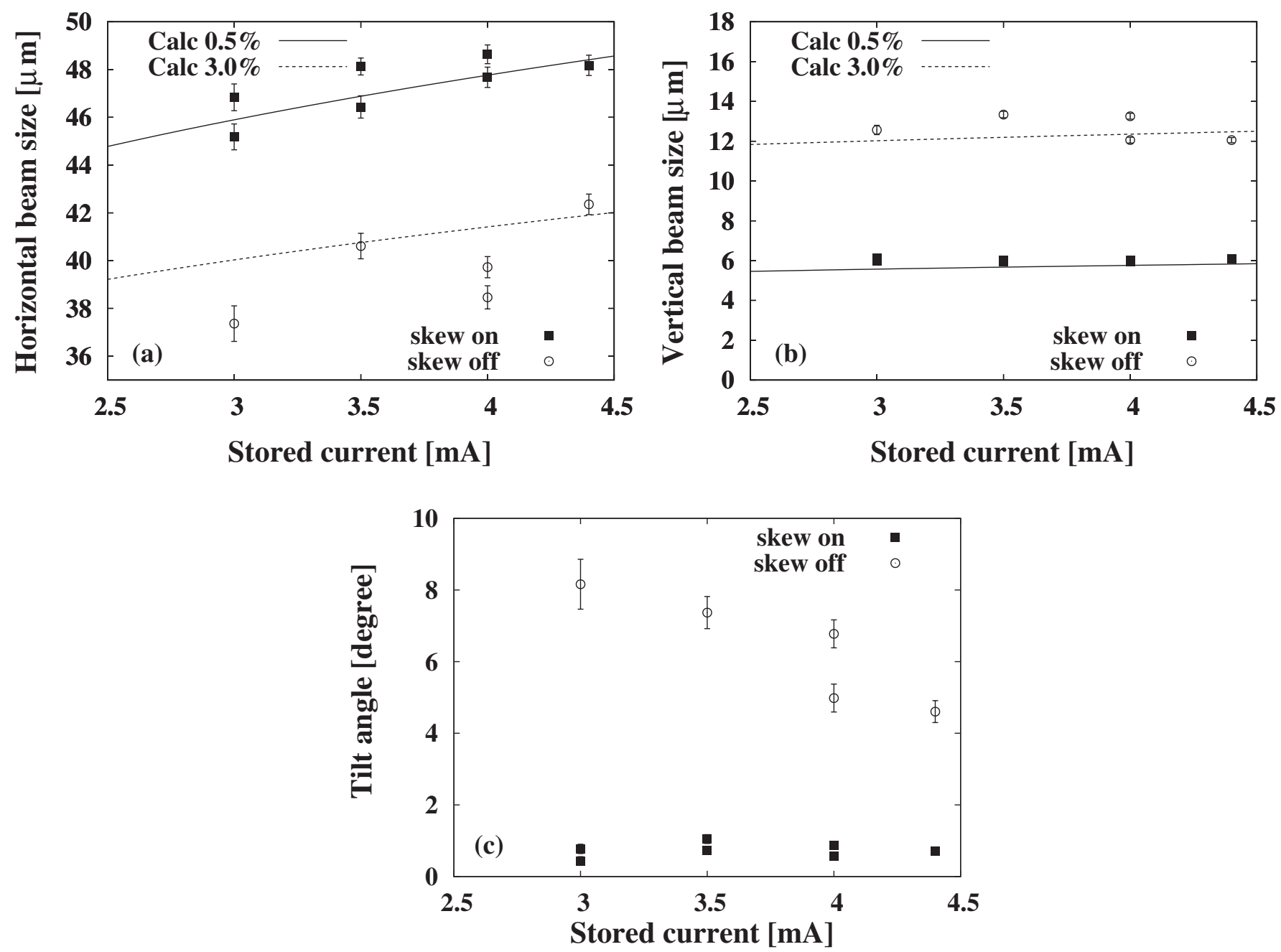

FIG. 21. Measured horizontal beam sizes (a) and vertical beam sizes (b) and tilt angles (c) vs stored current in the ATF damping ring. The solid boxes (open circles) show the data for the skew on (skew off) condition. The solid (dotted) lines of graph (a) and graph (b) are from calculations, assuming coupling ratios of $0.5 \%(3.0 \%)$. 
kinds of sextupole magnets. Figure 20 shows the typical beam profiles when a skew correction was carefully carried out [Fig. 20(a)] and all of the skew-quadrupole coils were turned off on purpose [Fig. 20(b)]. As shown in Fig. 20, we found that the vertical beam size increased and the measured beam profile was tilted when a skew correction was not applied. In order to measure the coupling dependence precisely, we measured the two sets of beam profiles at the same beam current when a skew correction was applied (hereafter called "skew on" condition), and the skewquadrupole coils were turned off (called "skew off" condition). Figure 21 shows all of the results of the two conditions of skew on and skew off. As shown in Fig. 21, the vertical beam sizes increased for all of the stored currents under the skew off condition compared with those under the skew on condition, while the horizontal one decreased. In order to estimate the coupling ratio, we also plot the calculation data including intrabeam scattering effect in Figs. 21(a) and 21(b). The data set of the skew on (skew off) condition agree with the calculation assuming the $0.5 \%(3.0 \%)$ coupling ratio. The absolute values of the measured tilt angles of the skew off condition are $(6 \pm$ 2) degrees, which is much larger than that of skew on condition of $(0.7 \pm 0.3)$ degrees.

\section{CONCLUSION}

In this paper, we have presented an improvement of the FZP monitor and measurement results of the ultralow emittance beam in the ATF damping ring under various conditions. First, by thermally disconnecting the Si crystal from the stepping motor, the position drift of the obtained image was drastically reduced by a factor of 100 , and fully stabilized within a few $\mu \mathrm{m}$ for one day. Second, we modified the FZP folder for a more precise beam-based alignment using $x$-ray SR. This avoids the effects of aberrations due to any misalignments of the FZPs. Third, the newly installed fast mechanical shutter allowed us to measure a beam image within $1 \mathrm{~ms}$. In addition, the x-ray CCD was synchronized with the beam-injection timing. We could measure the beam profile under the fully damped condition in the normal operation mode. At last, installation of the $\mathrm{x}$-ray pinhole mask system greatly reduced the background of $x$ rays passing through the MZP.

With the improved system, beam-profile measurements were performed on three days. By using a fast mechanical shutter, we could remove the effect of an unknown $100 \mathrm{~Hz}$ oscillation, which enlarged the measured vertical beam size, for the beam-profile measurement. We therefore could perform precise beam-profile measurements with a $1 \mathrm{~ms}$ shutter opening time. After carefully applying the skew correction, the measured horizontal beam sizes were about $50 \mu \mathrm{m}$, and the vertical beam sizes were about $6 \mu \mathrm{m}$ at above $3 \mathrm{~mA}$ stored current in the single-bunch mode, which corresponded to about $11 \mathrm{pmrad}$ of the vertical emittance. The measured beam sizes were in a good agree- ment with a calculation assuming coupling ratios of $(0.5 \pm$ $0.1) \%$. In addition, the measured energy spread also agreed well with the calculation. Thanks to the improved x-ray $\mathrm{CCD}$ and shorter time resolution of the newly installed fast mechanical shutter, we could also precisely measure the damping time of the ATF damping ring when the damping wigglers were turned on and off. The measurement results of the vertical damping ring agreed well with the design values. Furthermore, the coupling dependence of the beam profiles was obtained. Not only the horizontal and vertical beam sizes, but also the beam tilt angles, were measured precisely under the two coupling conditions. From these measurements, good performance of the improved monitor was confirmed.

Finally, we note that the improved FZP monitor is now routinely used and helps to produce and manipulate the ultralow emittance beam of the ATF damping ring during beam operation.

\section{ACKNOWLEDGMENTS}

First of all, we would like to express our gratitude to all members of the KEK-ATF group for their helpful support. We would like to thank Professor K. Ueda and Professor A. Kakizaki for their support and encouragement. We are also grateful to Professors Y. Totsuka, Y. Kamiya, S. Iwata, and A. Enomoto for their continuous support of this work. This research was partially supported by Grant-in-Aid Scientific Research (16540234) from the Ministry of Education, Culture, Sports, Science and Technology of Japan, and Joint Development Research at KEK.

[1] F. Hinode, S. Kawabata, H. Matsumoto, K. Oide, K. Takata, S. Takeda, and J. Urakawa, KEK Internal Report No. 95-4, 1995.

[2] Y. Honda et al., Phys. Rev. Lett. 92, 054802 (2004).

[3] T. Okugi, T. Hirose, H. Hayano, S. Kamada, K. Kubo, T. Naito, K. Oide, K. Takata, S. Takeda, N. Terunuma, N. Toge, J. Urakawa, S. Kashiwagi, M. Takano, D. McCormick, M. Minty, M. Ross, M. Woodley, F. Zimmermann, and J. Corlett, Phys. Rev. ST Accel. Beams 2, 022801 (1999).

[4] K. Kubo et al., Phys. Rev. Lett. 88, 194801 (2002).

[5] T. Mitsuhashi, Proceedings of the 1997 Particle Accelerator Conference, Vancouver, 1997, p. 766.

[6] H. Hayano, K. Kubo, T. Mitsuhashi, T. Naito, N. Terunuma, N. Toge, J. Urakawa, T. Okugi, and S. Kashiwagi, Proceedings of the 1999 Particle Accelerator Conference, New York, 1999), p. 2143.

[7] H. Sakai, Y. Honda, N. Sasao, S. Araki, Y. Higashi, T. Okugi, T. Taniguchi, J. Urakawa, and M. Takano, Phys. Rev. ST Accel. Beams 4, 022801 (2001).

[8] H. Sakai, Y. Honda, N. Sasao, S. Araki, H. Hayano, Y. Higashi, K. Kubo, T. Okugi, T. Taniguchi, N. Terunuma, J. Urakawa, and M. Takano, Phys. Rev. ST Accel. Beams 5, 122801 (2002). 
[9] Y. Honda, N. Sasao, S. Araki, H. Hayano, Y. Higashi, K. Kubo, T. Okugi, T. Taniguchi, N. Terunuma, J. Urakawa, Y. Yamazaki, K. Hirano, M. Nomura, M. Takano, and H. Sakai, Phys. Rev. ST Accel. Beams 6, 092802 (2003).

[10] Y. Honda, N. Sasao, S. Araki, Y. Higashi, T. Okugi, T. Taniguchi, J. Urakawa, Y. Yamazaki, K. Hirano, M. Nomura, M. Takano, and H. Sakai, Nucl. Instrum. Methods Phys. Res., Sect. A 538, 100 (2005).

[11] Z. Cai, B. Lai, W. Yun, E. Gluskin, D. Legnini, P. Ilinski, E. Trakhtenberg, S. Xu, W. Rodrigues, and H. Lee, Proceedings of the Tenth U.S. National Conference on Synchrotron Radiation Instrumentation, AIP Conference Proceedings 417 (American Institute of Physics, New York, 1997), p. 101.

[12] T. Weitkamp, O. Chubar, M. Drakopoulos, I. Snigireva, A. Snigirev, C. Schroer, F. Guenzler, and B. Lengeler, Proceedings of the 7th European Particle Accelerator Conference, Vienna, 2000, p. 1824.

[13] N. Nakamura, Y. Kamiya, T. Koseki, H. Takaki, N. Aoki, and K. Nakayama, Proceedings of the 2001 Particle Accelerator Conference, Chicago, 2001, p. 1628.

[14] K. Iida, N. Nakamura, H. Sakai, K. Shinoe, H. Takaki, M. Fujisawa, H. Hayano, M. Nomura, Y. Kamiya, T. Koseki, Y. Amamiya, N. Aoki, and K. Nakayama, Nucl. Instrum. Methods Phys. Res., Sect. A 506, 41 (2003).
[15] S. Takano, M. Masaki, and H. Ohkuma, Nucl. Instrum. Methods Phys. Res., Sect. A 556, 357 (2006).

[16] Recently, the $50 \mathrm{~nm}$ of the outermost zone width is available due to the progress of the nanofabrication technology in commerce.

[17] M. Fujisawa, H. Sakai, N. Nakamura, H. Hayano, and T. Muto, Proceedings of the Ninth International Conference on Synchrotron Radiation Instrumentation, AIP Conference Proceedings 879 (American Institute of Physics, Daegu, 2007), p. 1117.

[18] T. Muto et al. (to be published).

[19] K. Kubo, Phys. Rev. ST Accel. Beams 6, 092801 (2003).

[20] T. Naito, H. Hayano, Y. Honda, K. Kubo, M. Kuriki, S. Kuroda, T. Muto, N. Terunuma, J. Urakawa, H. Sakai, N. Nakamura, M. Korostelev, F. Zimmermann, and M. Ross, Proceedings of the 2005 Particle Accelerator Conference, Knoxville, 2005, p. 2809.

[21] SAD is a computer program for accelerator design (http:// acc-physics.kek.jp/SAD/sad.html).

[22] K. Kubo and K. Oide, Phys. Rev. ST Accel. Beams 4, 124401 (2001).

[23] K. L. F. Bane, H. Hayano, K. Kubo, T. Naito, T. Okugi, and J. Urakawa, Phys. Rev. ST Accel. Beams 5, 084403 (2002).

[24] P. Emma and T. Raubenheimier, Phys. Rev. ST Accel. Beams 4, 021001 (2001). 